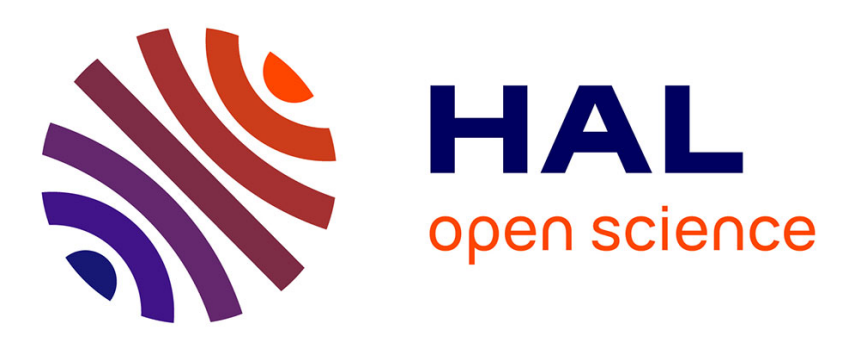

\title{
On the atomic structures, mobility and interactions of extended defects in GaN: dislocations, tilt and twin boundaries
}

A Bere, Anna Serra

\section{- To cite this version:}

A Bere, Anna Serra. On the atomic structures, mobility and interactions of extended defects in GaN: dislocations, tilt and twin boundaries. Philosophical Magazine, 2006, 86 (15), pp.2163-2196. 10.1080/14786430600640486 . hal-00513674

\section{HAL Id: hal-00513674 \\ https://hal.science/hal-00513674}

Submitted on 1 Sep 2010

HAL is a multi-disciplinary open access archive for the deposit and dissemination of scientific research documents, whether they are published or not. The documents may come from teaching and research institutions in France or abroad, or from public or private research centers.
L'archive ouverte pluridisciplinaire HAL, est destinée au dépôt et à la diffusion de documents scientifiques de niveau recherche, publiés ou non, émanant des établissements d'enseignement et de recherche français ou étrangers, des laboratoires publics ou privés. 


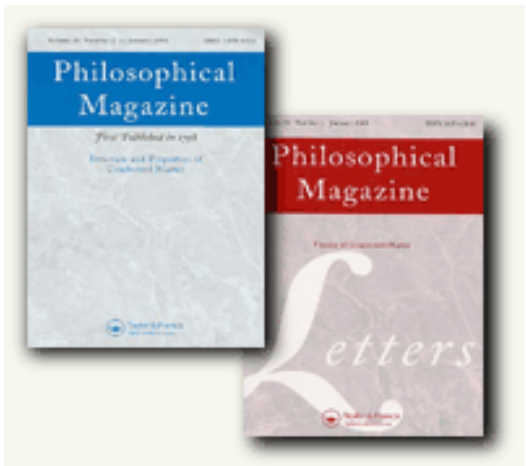

\section{On the atomic structures, mobility and interactions of extended defects in GaN: dislocations, tilt and twin boundaries}

\begin{tabular}{|c|c|}
\hline Journal: & Philosophical Magazine \& Philosophical Magazine Letters \\
\hline Manuscript ID: & TPHM-05-Apr-0128.R1 \\
\hline Journal Selection: & Philosophical Magazine \\
\hline $\begin{array}{r}\text { Date Submitted by the } \\
\text { Author: }\end{array}$ & 27-Jun-2005 \\
\hline Complete List of Authors: & $\begin{array}{l}\text { Bere, A; Université de Ouagadougouy, UFR/SEA/PHYSIQUE } \\
\text { Serra, Anna; Universitat Politecnica Catalonia, Matematica Aplicada } \\
\text { III }\end{array}$ \\
\hline Keywords: & $\begin{array}{l}\text { twinning, atomistic simulation, computer simulation, dislocations, } \\
\text { GaN, grain boundaries, lattice defects }\end{array}$ \\
\hline Keywords (user supplied): & \\
\hline
\end{tabular}

\section{S) ScholaroNE \\ Manuscript Central}


Results obtained by atomic computer simulation based on an adapted StillingerWeber (S-W) potential concerning the structure and relative stability of lattice dislocations, tilt and twin boundaries in $\mathrm{GaN}$ are discussed. The method used for the search and description of all possible atomic configurations depends on the crystallographic structure; consequently it is of general application and the results are transferable to the wurtzite binary compounds. On the contrary, the relaxed structures and their relative energetic stability are potential dependent. The results presented here correspond to a GaN model described by a pair potential. Whenever it has been possible our results have been compared with experiments or with ab initio calculations.

We present the core shape and energy of $\vec{a}$ and $\vec{c}$ crystal dislocations of both edge and screw character; [0001] tilt boundaries of misorientation angles from $9.3^{0}$ (corresponding to $\Sigma 37$ ) to $\theta=44.8^{\circ}$ (corresponding to $\Sigma 43$ ) and (10 $\overline{1} \mathrm{n}$ ) twin boundaries $(n=1,2,3)[1-4]$. The atomic structures of the tilt boundaries can be described in terms of the three stable structures of the prism $\vec{a}$-edge dislocation core. The (10 $\overline{1} 3)$ twin boundary is entirely described by 6-coordinated channels whereas the other twin boundaries present more complex structural units. 







\section{Introduction}

$\mathrm{GaN}$ is a semiconductor that has to be artificially produced. The growth has mainly been hetero-epitaxial on several foreign substrates with different orientations including a-plane $(1 \overline{1} 20)$, m-plane $(10 \overline{1} 0)$, r-plane $(01 \overline{1} 2)$ and c-plane (0001) (for a review see, e.g. Ref. [5]). The substrate employed determines the crystal orientation, polarity, polytype, the surface morphology, strain and the defects nature and concentration of the GaN film (for a review see, e.g. Ref. [6]). During the growing process a big amount of defects in the crystallographic structure are formed which will strongly influence the electronic properties of the GaN based devices. Therefore, a precise knowledge of their atomic and electronic structure is of main importance. These defects can be either localised or extended. The former, namely, point defects and their clusters can be studied with great accuracy by first principle calculations [7-11]. The later, need to be reproduced in systems formed by thousands of atoms and therefore their study is hardly affordable by ab initio calculations. The use of empirical effective potentials reduces enormously the number of calculations. This fact allows giving a preliminary overview to all possible configurations that can be later studied individually by first principles. Among the extended defects existing in the grown GaN, threading dislocations and tilt boundaries are well recognised by the experimental observations [12-14]. In particular, three types of threading dislocations are observed in epilayers grown on the substrate (0001) plane with Burgers vectors $1 / 3<1 \overline{2} 10>$ (a-type of edge character), [0001] (c-type of screw character) and 1/3 $<11 \overline{2} 3>$ (a+c type of mixed character). It has been found that, under certain techniques of growth, such as the two-step Epitaxial Lateral Overgrowth (2S-ELO) process [14], the dislocation line changes its orientation from [0001] to a direction lying on the basal plane. Thus, there is evidence of the creation of c-type dislocation with edge character and a-type dislocation with screw character during the growth process.

When the growth is produced on the (0001) surface of the substrate and the mosaic growth mode applies, individual islands rotated around the [0001] axis [15] are formed. The misorientation between these islands gives rise to the tilt boundary formation that is often associated with electronic states in covalent semiconductors.

In addition, the various epitaxial relationships, induces the formation of other extended defects, such as twins, that causes serious problems in the compound semiconductors as it reduces the single crystallite yield [16]. Whereas the most commonly observed twins in 
hexagonal-close-packed (h.c.p.) metals ( $\{10 \overline{1} 1\},\{10 \overline{1} 2\},\{11 \overline{2} 1\}$ and $\{11 \overline{2} 2\}$ twins) have been extensively studied [17-21], there is very little information on these twins in GaN. The only HREM published on twins in GaN to our knowledge concerns a cubic crystallite [22].

In this overview we present a study by computer simulation of the stable atomic structures of these defects. In the study of each defect there are two parts. First, all possible initial configurations that cannot be related by simple translations of one part of the crystal relative to the other are considered and described. This result is related to the wurtzite structure and can be used as starting point for the study of any binary compound. In section 2 there is a detailed description of the method used. Second, each particular configuration has been relaxed to find the minimum energy. The selection of the most stable configurations for a given defect by energetic criteria is done and only the structures of lower energy are considered. This result may be potential dependent; thus, the validity of the potential used has been checked by comparing the results with the experiment or with ab initio calculations. In section 2 there is a full description of the potential used.

\section{Computational method}

In this section we describe the characteristics of the simulated system, the interatomic potential used, the relaxation procedure and the method of analysis of the results.

\subsection{Construction of simulation cells}

The simulation cell is a set of atoms that contains the defect to be studied. This cell is surrounded by an external region (outer region) to avoid undesirable surface effects. The outer region has a thickness such that all inner-region atoms have a full neighbourhood within the range of the interatomic potential. The nature of the outer region depends on the defect. In the simplest case, i.e., point defects, the outer region is formed by fixed atoms located in sites of the perfect crystal. In the simulation of a dislocation, the system has to be infinite along the dislocation line. This is achieved by constructing a periodic system formed by the images of the cell along that direction. In this case the outer region in the other two directions is fixed. In the simulation of periodic interfaces, the outer region on the plane of the interface is periodic whereas in the perpendicular direction the outer region can be either fixed or periodic. 


\subsubsection{Atomic models for the grain boundary structures}


Consider two crystals, one designated white $(\lambda)$ and the other black $(\mu)$, with a fixed relative orientation represented by a matrix $\mathbf{P}$ that relates the black coordinate frame to the white. Assuming that individual atoms do not relax from their ideal black or white crystal sites, the structure of this dichromatic bicrystal depends on the chosen location of the interface plane and the relative position of the two crystals, the later being represented by $\mathbf{p}$ [24]. The interface plane can be chosen on the dichromatic pattern of the bicrystal [25] among the planes of the coincident site lattice (CSL). The CSL is characterized by the multiplicity $\Sigma$ (ratio between the volume of the unit cells of the CSL and the crystal lattice). For each interface, there is a misorientation angle between the bicrystals, $\theta$, which is defined as the angle between the $(11 \overline{2} 0)$ planes closest to the interface located on the upper and lower crystals respectively (see fig. 1 in [3]).

For the case of a periodic interface, each structure can be represented by a single point in a 2-D cell which is a Wigner-Seitz cell of the common translation vectors $\mathbf{p}$ parallel to the interface [26]. Vitek et al. [27] referred to such constructions as "cells of non-identical displacements" (cnid's), reflecting the fact that all structures obtained from a given reference structure by a displacement of the black crystal parallel to the interface can be represented by points within this cell. It was shown by Pond [25] that for the case of grain boundaries in materials with a single-atom basis, all of the possible bicrystal structures for a given relative orientation and interfacial orientation can be mapped into a single cnid. Thus, any pair of distinguishable structures represented by two distinct points in the associated cnid can be interrelated by the displacement $\Delta \mathbf{p}=(\Delta \mathrm{x}, \Delta \mathrm{y})$ corresponding to the vector joining the two mapping points. When the crystals exhibit $n$-atoms basis, the bottom surface of the upper crystal $\left(\mathrm{P}_{\lambda}\right)$ (assumed to be $\lambda$ ) can be terminated at $\mathrm{n}$ different levels thereby leading to $n$ distinguishable unrelaxed surfaces. The same situation arises to the $\mu$ crystal $\left(\mathrm{P}_{\mu}\right)$. It follows that a total multiplicity of $n x n$ distinguishable bicrystal structures can be created by bonding together all combinations of possible $\lambda$ and $\mu$ surfaces. For such bicrystals a set of nxn distinct cnid's exist. This number is reduced when the vectors $\Delta \mathbf{r}_{\mathbf{i j}}$ relating basis atoms $i$ and $j$ are parallel to the interface plane [28]. Thus, for a given misorientation and for a particular location of the interface, the total number of cnid's has to be identified and all the stable structures in each cnid have to be considered. In the GaN crystals of wurzite structure, with 4-atom basis, the maximum number of cnids is 16 .

In the case of the [0001] tilt boundaries, as the gallium and nitrogen atoms are aligned along the $c$ axis, the vector $\Delta \mathbf{r}_{\mathbf{G a}, \mathbf{N}}$ is parallel to the interface and each crystallographic plane has a 
maximum of two possible terminations, namely the two basal planes. Thus, the maximum number of different cnids is $2 \times 2$. For symmetrical tilt boundaries two of these cnids are interrelated by antisymmetry operators [28] and therefore only three cnids can be different. Also, for the special subset where all four atoms in the basis lie in the atomic plane parallel to the interface, there will be only one cnid. This is the case of one interface considered for

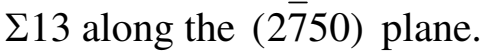

In the $[11 \overline{2} 0]$ twin boundaries, we have a total multiplicity of $4 \times 4$ bicrystal structures created by bonding together all of the combinations of possible $\lambda$ and $\mu$ surfaces. We recall that wurtzite structure can be imagined as two interpenetrating hexagonal substructures, one occupied by $\mathrm{Ga}$ atoms and the other by $\mathrm{N}$ atoms. The two substructures are shifted by approximately $3 / 8[0001]$, and the stacking sequence along [0001] is ...AaBbAaBb... where $\mathrm{Ga}$ atoms are in $\mathrm{A}$ and $\mathrm{B}$ planes and $\mathrm{N}$ atoms are in $\mathrm{a}$ and $\mathrm{b}$ planes. When the wurtzite structure is projected along [11 $\overline{2} 0$ ], each stacking corresponds to a row of one of the four atoms of the basis. Then, the four possible $\mathrm{P}_{\lambda} / \mathrm{P}_{\mu}$ bicrystal structures formed from the $\mathrm{A}(\lambda)$ surface are $\mathrm{A}(\lambda) / \mathrm{A}(\mu), \mathrm{A}(\lambda) / \mathrm{a}(\mu), \mathrm{A}(\lambda) / \mathrm{B}(\mu)$ and $\mathrm{A}(\lambda) / \mathrm{b}(\mu)$. Once the mirror twin is created, the other three are obtained by removing one, two or three $\mathrm{P}_{\mu}$ planes.

In our simulations the size of each crystallite depends on the period along each direction. Two periods were taken in the interface plane, i.e., perpendicular to [0001] (X-axis) and along [0001] (Z-axis). The number of periods in the direction normal to the interface (Y-axis) was chosen to ensure that there is no influence on the interface from the atoms of the outer region. Periodic boundary conditions were imposed, when possible, in the three directions. Special attention has to be paid in the $\mathrm{Y}$ direction, normal to the plane, to control if the same interface can be reproduced in the boundary [2]. Fig.1a shows schematically the studied interface A/B and the boundaries $\mathrm{C} / \mathrm{D}$ in the edges of the inner region. The condition for $\mathrm{A} / \mathrm{B}$ and $\mathrm{C} / \mathrm{D}$ to be equivalent is that it exists an antimirror symmetry perpendicular to the interface in the dichromatic complex. Fig.1b shows the couple A/B and C/D reproduced in the dichromatic complex of $\Sigma 7$ together with the antimirror plane. Most of the interfaces studied meet the requirement but the interface $(1 \overline{4} 30)$ of $\Sigma 13$ does not, and this should be considered in the calculation of the energy.

The point in the cnid that corresponds to the atomic structure of minimum energy was found by calculating the corresponding $\gamma$-surface. Thus, starting from a bicrystal obtained directly from the dichromatic complex, relative displacements parallel to the boundary where 
applied within the cnid and for every position the crystal was relaxed to the local minimum of energy.

\subsection{Empirical potentials describing atomic interactions in GaN}

Empirical potentials are constructed by considering that changes in the lattice energy can be written as a function of the position of the atoms comprising the material [29]. The energy function is based on different physical and symmetry criteria and constructed by fitting their parameters with experimental values of bulk properties and, if appropriated, some defects. It is important to notice that these parameters are adjusted for atoms already located in a particular structure; therefore these interatomic potentials describe, in fact, effective interactions. For particles with no net charge, the potentials are based on the idea that the force is repulsive if the atoms are very close and it is attractive vanishing smoothly to zero when the distance between them increases. The range of the potential - interaction distance - varies according to the kind of interaction.

When any empirical fitting is used to determine the parameters of the energy function, there is no uniqueness to the result: in most cases a number of quite different models can yield satisfactory agreement. In this work we have chosen the valence-force approach.

In the framework of the valence-force approach a set of interatomic potentials have been derived to study extended defects in GaN [1, 30-34].

Recently, a new analytical bond-order potential for $\mathrm{GaN}$ is available for the study of large systems: Nord et al [34] have presented a potential that describes structure and bonding of $\mathrm{Ga}, \mathrm{N}$ and $\mathrm{GaN}$ within an analytical form using a short-ranged bond-order algorithm. It consists of pair-like attractive and repulsive energy terms plus a bond angular term that models accurately the deformation of covalent bonds. The potential describes several solid structures of the pure elements and the compound; it has been used successfully to investigate native point defects.

The Keating potential [35] is the simplest one that has been used for the simulation of silicon, especially for zero temperature studies. A parameterisation has been proposed by Kim et al [30] to extend it for the simulation of GaN. These potentials have a strong limitation in the study of defects that comes from the necessity of having always four neighbours for each atom. 
In the scheme of the standard Stillinger and Weber potential [36], a series of potentials have been adapted to the defect study in III-V semiconductors [37]. This type of potential is a model comprising both two and three-atom contributions and accepts any atomic surrounding. GaN crystal has wurtzite structure and consists of atoms linked by strong and directional bonds. Thus, the potential energy function $\phi$ is approximated by a combination of pair and triplet potentials $\mathrm{V}_{2}$ and $\mathrm{V}_{3}$.

$$
\left\{\begin{array}{l}
\mathrm{V}_{2}\left(\mathrm{r}_{\mathrm{ij}}\right)=\varepsilon \mathrm{f}_{2}\left(\mathrm{r}_{\mathrm{ij}} / \sigma\right) \\
\mathrm{V}_{3}\left(\mathrm{r}_{\mathrm{i}}, \mathrm{r}_{\mathrm{j}}, \mathrm{r}_{\mathrm{k}}\right)=\varepsilon \mathrm{f}_{3}\left(\mathrm{r}_{\mathrm{i}} / \sigma, \mathrm{r}_{\mathrm{j}} / \sigma, \mathrm{r}_{\mathrm{k}} / \sigma\right)
\end{array}\right.
$$

where $\varepsilon$ is chosen to give $f_{2}$ depth -1 , and $\sigma$ is chosen to make $f_{2}\left(2^{\frac{1}{6}}\right)$ vanish.

The reduced pair potential was selected from the following 5-parameter family:

$f_{2}(r)=\left\{\begin{array}{l}A\left(B r^{-p}-r^{-q}\right) \exp \left[(r-a)^{-1}\right] \quad \text { if } r<a \\ 0 \quad \text { if } r \geq a\end{array}\right.$

where $\mathrm{A}, \mathrm{B}, \mathrm{p}$ and a are positive. This generic form automatically cuts off at $\mathrm{r}=\mathrm{a}$ without discontinuities in any $r$ derivatives. This fact is of main importance in most of the computer simulation applications. The three-body interaction is:

$$
\mathrm{f}_{3}\left(\mathrm{r}_{\mathrm{i}}, \mathrm{r}_{\mathrm{j}}, \mathrm{r}_{\mathrm{k}}\right)=\mathrm{h}\left(\mathrm{r}_{\mathrm{ij}}, \mathrm{r}_{\mathrm{ik}}, \theta_{\mathrm{jik}}\right)+\mathrm{h}\left(\mathrm{r}_{\mathrm{ji}}, \mathrm{r}_{\mathrm{jk}}, \theta_{\mathrm{ijk}}\right)+\mathrm{h}\left(\mathrm{r}_{\mathrm{ki}}, \mathrm{r}_{\mathrm{kj}}, \theta_{\mathrm{ikj}}\right)
$$

where $\theta_{\text {jik }}$ is the angle between $r_{j}$ and $r_{k}$ subtended at vertex $i$. The function $h$ belongs to a two-parameter family $(\lambda, \gamma>0)$ :

$$
\mathrm{h}\left(\mathrm{r}_{\mathrm{ij}}, \mathrm{r}_{\mathrm{ik}}, \theta_{\mathrm{jik}}\right)=\left\{\begin{array}{l}
\lambda \exp \left[\gamma\left(\mathrm{r}_{\mathrm{ij}}-\mathrm{a}\right)^{-1}+\gamma\left(\mathrm{r}_{\mathrm{ik}}-\mathrm{a}\right)^{-1}\right]\left(\cos \theta_{\mathrm{jik}}+1 / 3\right)^{2} \quad \text { if } \mathrm{r}<\mathrm{a} \\
0 \quad \text { if } \mathrm{r} \geq \mathrm{a}
\end{array}\right.
$$

Notice that the ideal tetrahedral angle is such that $\cos \theta=-1 / 3$, so that the potential favours pairs of bonds with the desired geometry. The values of the seven parameters were chosen to ensure that the diamond structure is the most stable at low pressure and that the liquid structure inferred from molecular dynamic simulations and the melting point are in good agreement with experiment.

Since the S-W potential, in standard form, could not reproduce the c/a ratio characteristic of the hexagonal structure, Béré et al [31] adapted the potential by adding a gaussian term to be able to include the third nearest neighbour interactions. A parameter that characterises the 
wurtzite structure of $\mathrm{GaN}$ is the displacement $\mathrm{u}$ along the $\mathrm{c}$ axis between the two sub-lattices. The gaussian parameters were chosen as the ones that minimise the energy of the perfect crystal by imposing the experimental values of c/a and $\mathrm{u}$. The derived potential was applied to investigate the atomic structures of two models of the $\{112 \overline{0}\}$ stacking fault with the starting configurations corresponding to the $1 / 6<2 \overline{2} 03>$ and $1 / 2<1 \overline{1} 01>$ displacement vectors [38]. Later, the radial (two body) and angular (three body) terms describing the energy of a GaN system were derived by Aichoune and co-workers [32] to take into account the specificity of the different bonds, namely, Ga-N, Ga-Ga and N-N. The fitting parameters were the lattice parameters, experimental elastic constants and the results of the ab initio calculations for an inversion domain boundary (IDB, described by the Holt model [39]) performed by Northrup and co-workers [40]. In fact, those authors claim that the IDB structure is quite unstable in $\mathrm{GaN}$, and would relax to another inversion domain boundary named IDB* having no wrong bonds. This structure can be formed by translating one side of an IDB by c/2 along the [0001] direction. Nevertheless, Potin and co-workers [41] reported an experimental evidence of the existence of both inversion domain boundaries. The parameters given by Aichoune and co-workers [32] are such that they only stabilise the IDB structure if the range of potential is fixed close to the first-neighbours distance (1nn).

We have generalized the applicability of Aichoune potential by slightly modifying the parameters (see table 1 and fig.2) and vanishing all interactions smoothly to zero just before the second-neighbours distance (2nn). No gaussian term is added to this generalization. The two body terms are plotted in Fig.2 as a function of each interaction type. The modified potential reproduces the lattice parameters and elastic constants; it stabilises the IDB structure and reproduces the ab initio results [40] obtained for the length of the wrong bond crossing the boundary and the energy per unit area. It gives the IDB* as the most stable configuration although it overestimates its energy. The potential is able to discriminate two close minima of similar energy corresponding to two configurations of the prism edge dislocation and gives results that may well compare with ab initio calculations as described in section 3. Among the factors contributing to the inaccuracy of the potential there is the fact that such a potential cannot accurately describe radial and angular deformations simultaneously [42] and it does not consider explicitly the electronic effects. Moreover, being an empirical potential, it is affected by the experimental error assigned to the fitting parameters. Even though, the comparison with the ab initio results indicates that the S-W potential gives a reasonable approximation of the energies and thus, S-W is a suitable 


\section{RESULTS}

Atomic structure and energy of dislocations

\subsubsection{Characterization of the dislocation core}

Two complementary characterizations have been applied to analyse the dislocation cores. The first one, based on the behaviour of the strain energy stored in the cylinder of radius $\mathrm{R}$ containing the dislocation line, determines the core radius and the core energy.

The strain energy of a dislocation within the linear elasticity theory is given by [23]: $\mathrm{E}=\mathrm{A}_{\mathrm{th}} \ln \left(\mathrm{R} / \mathrm{r}_{\mathrm{c}}\right)$, where $\mathrm{r}_{\mathrm{c}}$ is the dislocation core radius and $\mathrm{R}$ is the radius of a cylinder containing the dislocation line. The pre-logarithmic factor $A_{t h}$ is $\frac{\mu b^{2}}{4 \pi(1-v)}$ for the edge 
dislocation and $\frac{\mu b^{2}}{4 \pi}$ for the screw, where $b$ is the Burgers vector, $\mu$ is the shear modulus and $v(0.37$ for $\mathrm{GaN}[44])$ is the Poisson ratio. The expression of the energy E does not include the core energy of the dislocations, where the linear elasticity theory cannot be applied.

Thus by plotting the energy of a simulated dislocation versus $\ln (\mathrm{R})$ it is possible to identify the core radius as the value from which the curve starts being linear. The slope of the linear part gives the value of the pre-logarithmic factor, $A_{0}$, which takes into account the anisotropy of the crystal. The values of $\mathrm{A}_{0}$ can be compared to the theoretical values (See table 2).

The second characterization is based on the map of differential displacements of neighbouring pairs of atoms [45] that gives information on the shape and extension of the dislocation core. The components of the difference in displacement $\Delta \mathbf{u}$ between neighbouring atoms, measured from a suitable reference state, are calculated and plotted in the form of arrows superimposed on the atomic projection, the arrow length denoting the difference between $\Delta \mathrm{u}_{\mathrm{i}}$ and the components of an appropriate translation vector.

\subsubsection{Dislocations with $b=1 / 3<11 \overline{2} 0>$}

\subsubsection{Edge dislocation}

This edge dislocation lies along [0001] and its glide plane is the $\{1 \overline{1} 00\}$ plane. The core presents three stable configurations that can be related to the location of the origin of displacements imposed to the atoms to create the dislocation in the initial configuration.

Fig. 3a shows the relaxed core configuration of the dislocation with line dislocation origin located between two closely spaced $(10 \overline{1} 0)$ planes. This core presents a $5 / 7$-coordinated channel structure $(5 / 7$ core $)$ with wrong bonds. Fig $3 b$ shows the map of differential displacements of neighbouring pairs of atoms [45] and demonstrates that the core is spread about 5a lattice parameters along the two adjacent $(10 \overline{1} 0)$ planes that contain the 5/7coordinated channel.

Figs $4 \mathrm{a}$ and 5a show the core structures of 4 and 8-coordinated channels (4-core and 8-core) corresponding to the location of the line dislocation between two widely spaced (10 $\overline{1} 0)$ planes. The 4-core is characterised by four bonds practically in the same plane and the 8core has a dangling bond. Figs $4 \mathrm{~b}$ and $5 \mathrm{~b}$ show that both structures have quite comparable 
displacement maps. The cores spread mainly in one (10 $\overline{1} 0)$ plane although displacements up to $5 \%$ of the Burgers vector length are distributed over the two adjacent planes in a region of about 7 a lattice parameters.

Fig.6 shows the energy $E_{s}$ stored in a cylinder of radius $R$ as a function of $\ln (R)$ for the case of the $\vec{a}$ dislocation in a relaxable region of $46 \AA$ of radius. For the $5 / 7$-core the slope is $A_{0}$ $=0.78 \mathrm{eV} / \AA$ and the curve starts being linear from $\ln (\mathrm{R} / \AA)=1.9$ which gives a core radius of $6.7 \AA$ and a core energy of $1.46 \mathrm{eV} / \AA$.

Similar studies of the total energy $\left(E_{\mathrm{s}}\right)$ against $\ln (\mathrm{R})$ have been done with the other two stable cores. The slope and the core radius for both configurations were found to be the same than for the $5 / 7$ structure, as expected. However, the core energy $(E c=1.72 \mathrm{eV} / \AA)$ is $3 \%$ higher than that obtained for the 5/7-structure core energy.

According to our calculations the 5/7 core is the most stable followed by the 4 and 8 cores. The SW potential is sensitive to the two minima of energy corresponding to the 4 and 8 cores although the difference in their energies is within the inaccuracy of the potential. These two cores are related by a conservative movement of the dislocation line along the glide plane, whereas the 4 and 8 cores are related to the 5/7 core by a climb of the dislocation line.

The existence of these three cores has been proved experimentally by HREM [12] and by Zcontrast [46]. No other core structures have been observed.

\subsubsection{Screw dislocation}

The dislocation core presents two stable configurations related to the origin of the displacements imposed to the atoms when the dislocation is introduced. The displacement maps are presented in figs. 7. In the two cases there are displacements in both basal and prism planes showing dislocation cores extended within a cylinder of a diameter of about $4 \mathrm{a}$ lattice parameters. The main difference between the two cores is in the displacements along the prism plane. One core is spread on one prism plane whereas the other is distributed in two prism planes.

In Fig. 6, it is also shown the corresponding function of energy versus $\ln (\mathrm{R})$ calculated in a relaxable region of a radius of $46 \AA$ for the $\mathrm{R} 2$ core. The slope is $\mathrm{A}_{0}=0.59 \mathrm{eV} / \AA$ and the curve starts being linear from $\ln (\mathrm{R})=1.9$ which gives a core radius of $6.7 \AA$ and a core energy of $1.2 \mathrm{eV} / \AA$. 


\subsubsection{Dislocations with $b=[0001]$}

\subsubsection{Edge dislocation}

The $\overrightarrow{\mathrm{c}}$ edge dislocation has a $\{10 \overline{1} 0\}$ glide plane and lies along the [1 $\overline{2} 10]$ direction. The dislocation line can be located either between two widely spaced (10 $\overline{1} 0)$ planes (position S1) or between two closely spaced planes (position S2). In the former interplanar space each $\mathrm{Ga}$ atom of one plane is linked to one $\mathrm{N}$ atom of the other plane. In the later, each $\mathrm{Ga}$ atom of one plane is linked to two $\mathrm{N}$ atoms of the other plane.

The stable core configurations are shown in Figs 8 and 9. The core shown in fig. 8 has been obtained locating the dislocation line in the position S1. The map of relative displacements shows that the core is spread on two adjacent prism planes along a distance of 4 Burgers vectors. This core has lower energy than the core shown in fig. 9 that has been obtained locating the dislocation line in the position S2. The map of relative displacements shows that the core is extended to three prism planes and spread along 4 Burgers vectors. The core radius deduced for this configuration is $8.5 \AA$ and its core energy is $3.68 \mathrm{eV} / \AA$.

\subsubsection{Screw dislocation}

Fig. 10a shows the displacement map for the core obtained when the dislocation line is in a plane of mirror glide symmetry of the crystal (position T1), and then the displacements almost keep such symmetry. Such map presents a core located inside a cylinder of radius $2 \mathrm{a}$ $\approx 6.4 \AA$. This configuration presents slightly smaller energy than the second stable configuration that is obtained by locating the dislocation line in coincidence with the threefold axis (position T2). of the crystal (fig.10b). The corresponding displacement map shows a core constrained into a cylinder of radius $1.5 \mathrm{a} \approx 4.8 \AA$. From the curve of the strain energy, we deduce the slope $\mathrm{A}_{0}=1.35 \mathrm{eV} / \mathrm{A}$, a core radius of $6.7 \AA$ and a core energy of $2.82 \mathrm{eV} / \AA$.

The core radius, core energies and pre-logarithmic factors of the studied dislocations are summarized in table 3. 


\subsubsection{Validation of the $S$-W empirical potential in the simulation of extended defects}

As mentioned in section 2.2, the S-W potential used was derived to reproduce the inversion domain boundaries found experimentally. In this section we use the results on dislocation energies as a test on the applicability of the potential to the study of extended defects.

We have compared our calculations of the dislocation energies with some results reported in the literature obtained by ab initio methods (see table 3 ). The fact that the ab initio results have been obtained in crystals of smaller sizes than in the present calculation implies that, although the calculations are more precise, the systems are often not fully relaxed. In order to compare the results we have calculated the energy of the dislocations within cylinders of the same radius as the ones used in the ab initio calculations, i.e., $7.5 \AA$ and $8.5 \AA$.

\section{Prism screw dislocation}

In the present simulation, when the total relaxable region has a radius of $46 \AA$, the energy inside a cylinder of $7.5 \AA$ is about $2.9 \mathrm{eV} / \AA$ but if the relaxable region is constrained to the cylinder of a radius of $7.5 \AA$, then the energy is $3.3 \mathrm{eV} / \AA$. The decrease of $0.4 \mathrm{eV} / \AA$ by increasing the size of the simulated crystal is directly related to the long-range relaxation. The value of $3.3 \mathrm{eV} / \AA$ compares with $4.0 \mathrm{eV} / \AA$ (in a cylinder of radius $7.5 \AA$ ) obtained by Northrup [10] and $4.88 \mathrm{eV} / \AA$ (in a cylinder of radius $8.5 \AA$ ) obtained by Elsner and coworkers [8], both using first principle calculations.

\section{Prism edge dislocation.}

First principle calculations [8] performed with a supercell containing the 8-atom ring core gives an energy stored in a cylinder of radius equal to $8.5 \AA$ of $2.19 \mathrm{eV} / \AA$. The energy per unit length of this configuration for the same radius with the $\mathrm{S}-\mathrm{W}$ potential is $1.95 \mathrm{eV} / \AA$. The difference of $0.24 \mathrm{eV} / \AA$ can be partly due to the bigger system (better relaxation) considered in the S-W model. However, since this value is of the same order of the difference in energies between the 5/7-core and 8-core, it indicates that the $\mathrm{S}-\mathrm{W}$ model cannot discriminate which is the core of lower energy. In fact, Wright and Grossner [9] have calculated the formation energy of the $5 / 7$ and 8 cores and have shown that they 
exhibit a pronounced dependence of the Fermi level. They report that the 8-core has an energy lower than the 5/7-core in the neutral charge state. However recent Density Functional based Tight Binding (DFTB) simulations [49] present the 5/7-core structure as the most stable configuration in coincidence with our results.

\subsection{Atomic structure and energy of tilt boundaries}

A variety of [0001] tilt boundaries with misorientation angles from $\theta=9.3^{\circ}$ (corresponding to $\Sigma 37$ ) to $\theta=44.8^{\circ}$ (corresponding to $\Sigma 43$ ) have been simulated $[2-3,51]$. There areexperimental images of all the boundaries studied, some of them have been found in $\mathrm{GaN}$ [12] and the others in materials of wurtzite structure [52].

As a general trend, all structural units can be related to the structures of the cores of the $\vec{a}$ edge dislocation. We notice that whereas the dislocation cores depend on the location of the dislocation line, the configurations of the interfaces depend on the chosen CNID.

Lets consider, as examples, the periodic boundaries determined respectively by $(3 \overline{5} 20)_{\lambda} /(2 \overline{5} 30)_{\mu}$ in the $\Sigma 19$ (misorientation angle of $\left.13.4^{0}\right)$ and by $(2 \overline{3} 10)_{\lambda} /(1 \overline{3} 20)_{\mu}$ in the $\Sigma 7$ (misorientation angle of $21.6^{\circ}$ ); figs 11 and 12 respectively.

In both cases the $\gamma$-surface study reveals two different structures (S1 and S2) of low energy related to two different cnids $(\mathrm{C} 1$ and $\mathrm{C} 2)$. $\mathrm{C} 1$ is obtained by facing a-type atoms of the white $(\lambda)$ crystal to b-type atoms of the black crystal (shown in figure 11 for the $\Sigma 19$ ) and $\mathrm{C} 2$ is obtained by suppressing from $\mathrm{C} 1$ the a-type atoms of the white crystal. S1 (figs.11a and 12 a) present 5/7 coordinated channels that compares with the 5/7 edge dislocation core (fig.3a). These boundaries have been observed experimentally [12]. S2 (figs.11b and 12b) presents 4 coordinated channels like the edge dislocation core in fig.4a. Initially, in the starting configurations of the four results presented, the two half crystals are relatively displaced along $\vec{c}$ by $\Delta \mathrm{x}=0.375$ c so that during relaxation the bonds can be restored, i.e., the $5 / 7$ dislocation core is reproduced and the region of the interface between the 4 coordinated channels relaxes to an almost perfect structure. In agreement with the dislocation energies, the surface energy of the configuration $S 1\left(E_{S 1}=1518 \mathrm{~mJ} / \mathrm{m}^{2}\right.$ for the $\Sigma 19$ and $E_{\mathrm{S} 1}=1827 \mathrm{~mJ} / \mathrm{m}^{2}$ for the $\left.\Sigma 7\right)$ is smaller than the surface energy of S2 $\left(E_{\mathrm{S} 2}=1806\right.$ $\mathrm{mJ} / \mathrm{m}^{2}$ for the $\Sigma 19$ and $\mathrm{E}_{\mathrm{S} 2}=2357 \mathrm{~mJ} / \mathrm{m}^{2}$ for the $\Sigma 7$ ). There are other configurations in $\mathrm{C} 1$ (fig. 11c and 11d) related to $\mathrm{S} 1$ that correspond to translation states of the $\mathrm{S} 1$ structure. They present $5 / 7$ channels of alternate orientations more densely distributed than in $\mathrm{S} 1$. The 
energy of these configurations for the $\Sigma 19$ interface is $1921 \mathrm{~mJ} / \mathrm{m}^{2}$ for the structure in Fig. $11 \mathrm{c}$ and $2452 \mathrm{~mJ} / \mathrm{m}^{2}$ for the structure in Fig.11d respectively.

The $\Sigma 37$ interface with a misorientation angle of $9.4^{0}$ presents qualitatively the same structures S1 and S2 described above, the only difference is that the coordinated channels are less densely distributed along the interface because the misorientation to be accommodated is smaller. As a consequence the energy is smaller, i.e., $E_{\mathrm{S} 1}=1286 \mathrm{~mJ} / \mathrm{m}^{2}$ and $\mathrm{E}_{\mathrm{S} 2}=1485 \mathrm{~mJ} / \mathrm{m}^{2}$.

It can be seen in the figures 11 to 12 that the structural unit of the configurations of low energy is formed by a single coordinated channel. This is not a general characteristic of the [0001] tilt boundaries. It follows the description of some boundaries where the structural unit is formed by two or more coordinated channels. The relaxed configuration of a $\Sigma 43$ boundary along the $(1 \overline{7} 60)$ plane has an energy of $E_{\mathrm{S} 2}=1834 \mathrm{~mJ} / \mathrm{m}^{2}$ and it is formed by units of two 5/7 coordinated channels (fig.13). The relaxed configuration of $\Sigma 49$ along $(3 \overline{8} 50)$ is shown in fig. 14 . It has an energy of $E_{S 2}=1692 \mathrm{~mJ} / \mathrm{m}^{2}$ and it is formed by units of one 5/7 plus one 4 coordinated channel equally spaced.

$\Sigma 13$ along the plane $(2 \overline{7} 50)$, gives an example of an interface that has only one CNID since there are atoms of both the a- type and b-type in the plane. This boundary presents a configuration formed by three types of coordinated channels (see fig.15a) although the configuration of lowest energy $\left(E_{\mathrm{S} 1}=2185 \mathrm{~mJ} / \mathrm{m}^{2}\right)$ is formed by three $5 / 7$ channels as shown in fig.15b. It has been observed experimentally [50] in $\mathrm{ZnO}$ that has the wurtzite structure as $\mathrm{GaN}$.

Finally in fig. 16 we present a second interface of $\Sigma 13$ along $(1 \overline{4} 30)$ plane that corresponds to the case mentioned is section 2 where the plane perpendicular to the interface is not an antimirror plane of the dichromatic pattern. The structure of lower energy of the first interface $(\mathrm{A} / \mathrm{B})$ is presented in fig.16a. This configuration has an energy of $\mathrm{E}_{\mathrm{I}}=1528$ $\mathrm{mJ} / \mathrm{m}^{2}$. It exhibits two $5 / 7$ channels of alternate orientations and has been observed experimentally [51]. The structure of the second interface (C/D) is formed by one 5/7 and one 4-coordinated channels as shown in fig. 16b. Its energy $E_{\mathrm{I} 2}=2659 \mathrm{~mJ} / \mathrm{m}^{2}$ is higher than the energy of the first interface.

The crystallographic parameters and energies of [0001] tilt boundaries are summarised in table 4. We notice that, for a given [0001] tilt boundary, the energy of a configuration is related to the type of $\vec{a}$ edge dislocation that appears on the structural unit. Therefore the 
sensitivity of the potential to the discrimination of the 4 and 5/7 dislocation cores is of main importance.

\subsection{Dislocations mobility and boundary-dislocation interaction}

\subsubsection{Glide of $1 / 3[2 \overline{1} \overline{1} 0]$ edge dislocations}

The glide plane of $\vec{a}$ edge dislocations is the $(01 \overline{1} 0)$ plane. As we mentioned in the introduction, the atomic structure of the dislocation core depends on the location of the dislocation line and this has direct influence on the mobility of these dislocations too. In fact the $\mathrm{C}_{5 / 7}$ core, located between the narrowly spaced $\{01 \overline{1} 0\}$ planes, was immobile under strains up to $5 \%$. The energies of the $\mathrm{C}_{4}$ and $\mathrm{C}_{8}$ cores have close values $(1.72 \mathrm{eV} / \AA$ in a cylinder of radius $\mathrm{R}=6.7 \AA$ ) and represent two minima separated by a low energy barrier (table 3). This characteristics favour the glide movement of the dislocation. In fact, the dislocation moves under a critical strain of $1 \%$ and the core transforms as $\mathrm{C}_{4}, \mathrm{C}_{8}, \mathrm{C}_{4}, \ldots$ The shape and extension of the dislocation core can be identified by means of the map of differential displacements (MDD) of neighbouring pairs of atoms [45]. This map characterizes the different cores and has been used here to follow the movement of the dislocations. In figs. 4 and 5 there are two MDD corresponding to a $\mathrm{C}_{4}$ and $\mathrm{C}_{8}$ cores respectively showing that they are essentially spread along the glide plane.

\subsubsection{Boundary movement and boundary interaction with the $\vec{a}$ edge dislocation}

We have restricted the mobility study to the boundaries of low misorientation formed by $\mathrm{C}_{4}$ dislocation cores. We present the case of the $\Sigma 37$ boundary. On the one hand, we checked if the $\mathrm{C}_{4}$ units in the boundaries still behave as dislocations, for instance, keeping their mobility. On the other hand, we studied the feasibility of the incorporation of a crystal dislocation to an existing boundary and the interaction dislocation-boundary.

The system was formed by a bicrystal containing a low angle boundary. The relaxable region contained two $\mathrm{C}_{4}$ cores bounded by fixed $\mathrm{C}_{4}$ cores at both sides; the distance between cores, i.e., the period of the boundary, is $T=\sqrt{3} 7 \mathrm{a}$. To induce the movement of the dislocations we applied a shear strain perpendicular to the boundary. The reorganization of the atoms of the boundary was investigated by comparing the strained and unstrained 
boundaries through the MDD. fig. 17 shows the MDD of the two relaxable $\mathrm{C}_{4}$ cores displaced to the left and right respectively, as a result of a shear strain of $\pm 4 \%$. The dotted line indicates the location of the initial boundary. The movement of these $\mathrm{C}_{4}$ units and the fact that for this boundary the relation for low angle boundaries, i.e., $\operatorname{tg} \alpha=\frac{|\mathrm{b}|}{|\mathrm{T}|}$, is accomplished allow us to identify the structural units of the boundary as being crystal dislocations. Under a perpendicular shear stress, the boundary bends and eventually the dislocation can leave it. The opposite process, i.e., the incorporation of a crystal dislocation to the boundary submitted to a local stress happens if the glide plane of the crystal dislocation does not coincide with the glide plane of the structural units of the boundary. Fig.18 shows the different reactions that may occur depending on the glide plane and sign of the interacting dislocation. The dislocations were placed near the boundary and the system was relaxed, no strain was applied. In all cases the expected interaction between two edge dislocations is accomplished. Fig.18a shows the repulsion when the crystal dislocation is located just beside the $\mathrm{C}_{4}$ unit of the boundary. Both dislocations move in opposite directions about $6 \mathrm{a}_{0}\left(\mathrm{a}_{0}\right.$ is the lattice parameter), thus the crystal dislocation pushes the $\mathrm{C}_{4}$ out of the boundary. If the sign of the dislocation is reversed there is a total recombination and the boundary loses the corresponding structural unit as shown in fig.18c. Fig. $18 \mathrm{~b}$ corresponds to an intermediate case where the crystal dislocation was located on a glide plane between two structural units. The stress field of the boundary is short ranged and the dislocation is in equilibrium at distances larger than $2 \mathrm{a}_{0}$. Fig.19 shows that, under a perpendicular shear strain of $2 \%$, the dislocation joins the boundary. If the strain is of opposite sign the two nearest structural units of the boundary move until they are aligned with the crystal dislocation. Notice that the lower dislocation core has transformed from $\mathrm{C}_{4}$ to $\mathrm{C}_{8}$ core. Since the remaining part of the boundary has been frozen during the relaxation process, the final configuration is a stepped boundary. The same configuration would occur in a pinned boundary under strain.

\subsection{Atomic structure and energy of twin boundaries}

Three twin boundaries, namely, $(10 \overline{1} 1),(10 \overline{1} 2)$ and $(10 \overline{1} 3)$ twins have been simulated. For each boundary we have considered distinct reference bicrystal structures and we have calculated the corresponding $\gamma$-surfaces to search all possible stable configurations. The 
core structure of the twin boundaries was found to be dependent on the starting bicrystal structure $\mathrm{P}_{\lambda} / \mathrm{P}_{\mu}$ (see subsection 2.1.2). Although some starting configurations do not present an entirely symmetrical shape the mirror symmetry was recovered by rigid body displacements parallel to the twin plane and/or during the relaxation process. 4-, 8- or 5/7coordianted channels separated by 6-coordinnated channels describe the $(10 \overline{1} 2)$ boundary whereas 6-coordinated channels uniquely composed the $(10 \overline{1} 3)$ boundary that is the one with lowest energy. The energy calculations show that the low-energy structures only exhibits a pure mirror symmetry for the $(10 \overline{1} 2)$ and (10 $\overline{1} 3)$ twins.

\section{4 .1 (10̄11) Twin}

Three relaxed structures of this twin projected along [11 $\overline{2} 0]$ are shown in fig.20. The four symbols represent the four atoms of the basis that are located in four layers along the normal to the twinning plane. The two first structures, $S_{1}$ (fig.20a) and $S_{2}$ (fig.20b), were obtained with an $\mathrm{A}(\lambda) / \mathrm{a}(\mu)$ bicrystal configuration and the latter, $S_{3}$ (fig.20c), from a $\mathrm{B}(\lambda) / \mathrm{B}(\mu)$ one. A single plane of atoms formed by the coalescence of two $(10 \overline{1} 1)$ planes forms the boundary of the structure $J_{1}$ whereas a corrugated plane marks the structures $J_{2}$ and $J_{3}$. The structure $J_{2}$ is related to the structure $J_{1}$ by a rigid body displacement in the direction of the twin plane with a clear effect on the mirror symmetry by changing the motif in one of the crystals. Our energetic calculations shown that structures characterized by corrugated boundary plane $\left(\mathrm{J}_{2}\right.$ and $\left.\mathrm{J}_{3}\right)$ are energetically more favourable than the structure with a single plane boundary $\left(\mathrm{J}_{1}\right)$. These energies are $2709 \mathrm{~mJ} / \mathrm{m}^{2}$ for the structure $\mathrm{J}_{3}, 2753$ $\mathrm{mJ} / \mathrm{m}^{2}$ for the structure $J_{2}$ and $3567 \mathrm{~mJ} / \mathrm{m}^{2}$ for the structure $J_{1}$. Other stable boundaries were found but the twin energy was higher than the low-energy structure given above.

\subsection{2 (10 12$)$ Twin}

We present in fig.21 the three atomic structures of low energy. The $\mathrm{K}_{1}$ configuration contains a 4-coordinated channel (4-interface, Fig. 21a), the $\mathrm{K}_{2}$ one, an 8-coordinated channel (8-interface, Fig.21b) and the $\mathrm{K}_{3}$ configuration, a 5/7-coordinated channel (5/7interface, Fig. 21c). The structures were obtained from a twin with mirror symmetry by removing one, two or three $(10 \overline{1} 2)$ layers of atoms. Then, the mirror symmetry was recovered in the relaxation process. All these boundaries are marked by a single plane of 


\section{$2.4 .3 \quad(10 \overline{1} 3)$ Twin}

Figure 22 shows the low-energy structure obtained for this twin. A single plane of atoms formed by the coalescence of two (1013) planes as in the previous cases marks the boundary plane. The relaxed structure was obtained from an $A(\lambda) / B(\mu)$ bicrystal configuration. It shows mirror symmetry and the structural units composing this boundary exhibit six-coordinated channels. The twin-boundary energy is $1302 \mathrm{~mJ} / \mathrm{m}^{2}$. Other stable boundaries were found but the twin-boundary energy was much higher. This energy is much lower than the values obtained for the low-energy structures of the $(10 \overline{1} 1)$ and $(10 \overline{1} 2)$ twins. This is in agreement with the fact that the 6-coordinated channels minimize the distortion of the tetra-coordination.

\section{Summary and conclusions}

Extended defects appearing in GaN films grown on substrates of hexagonal structure have been studied by means of atomic computer simulation.

In this work we describe $\vec{a}$ and $\vec{c}$ dislocations of both edge and screw character. Among the two dimensional defects we have focussed on both the periodic [0001] tilt boundaries and $(10 \overline{1} \mathrm{n})$ twin boundaries that, together with stacking faults and inversion domains, are the main interfaces found in grown $\mathrm{GaN}$. 
The atomic interactions are represented by an empirical potential of the Stillinger - Weber form adapted to simulate the three types of interactions $\mathrm{Ga}-\mathrm{Ga}, \mathrm{N}-\mathrm{N}$ and $\mathrm{Ga}-\mathrm{N}$. This potential was fitted to reproduce the inversion domains found in $\mathrm{GaN}$. In this work we validate the potential by comparing our results on the energy of dislocation cores with first principle calculations.

The atomic structure of a dislocation core depends on the location of the dislocation line. This is clearly seen in the case of the $\vec{a}$ edge dislocation where the dislocation line can be located either between two widely spaced (10 $\overline{1} 0)$ planes or between two closely spaced ones. As a result three distinct cores appear, the 5/7 channel related to the narrow space and the 4 channel and 8 channel related to the wide space. In the later case the dislocation core transforms from 4 channel to 8 channel by a conservative glide of the dislocation line. The transformation of the $5 / 7$ channel either to the 4 channel or 8 channel can be done only by climb. The displacement maps of the cores show that the 4 and 8 cores are spread on the glide plane, whereas the $5 / 7$ core is more localised but it is distributed on two adjacent planes. The comparison of the corresponding maps indicates a better mobility of the 4 and 8 cores than the 5/7 core that may be sessile. These atomic structures are the only ones observed experimentally and in our study we found that no other stable structures of low energy exist. The $\vec{a}$ screw presents two stable cores with displacement maps quite extended indicating that there are no preferential glide planes.

In the case of the $\overrightarrow{\mathrm{c}}$ screw threading dislocation the displacement map shows a three-fold symmetry that is kept in the open cores found experimentally. For completeness we present the results for the $\vec{c}$ edge dislocation. The core presents high distortion in bond length and angles.

We present a set of [0001] tilt boundaries with misorientations from $9.4^{0}$ to $44.8^{0}$. We found that the stable structural units, in all cases, are formed by combinations of the same $5 / 7,4$ and 8 channels as in the $\vec{a}$ edge dislocation cores. In general, there are two different possible structures that cannot be related by a translation parallel to the interface. In the boundaries of small rotation angle the structural unit of smaller energy is formed either by one 5/7 channel or by one 4 channel. These boundaries can be considered, indeed, as formed by aligned $\vec{a}$ edge dislocations. The energy of the boundaries is a function of the density of dislocations that form the boundary but there is no direct relation between the angle of misorientation and the energy. 
The mobility of the a-edge dislocations has been investigated and it was found that the $\mathrm{C}_{5 / 7}$ core is sessile while the $\mathrm{C}_{4}$ and $\mathrm{C}_{8}$ cores are mobile. During the movement the cores change from $\mathrm{C}_{4}$ to $\mathrm{C}_{8}$ to $\mathrm{C}_{4} \ldots$ This result is in agreement with the map of differential displacements since $\mathrm{C}_{4}$ and $\mathrm{C}_{8}$ cores have a map essentially spread on the glide plane whereas the $\mathrm{C}_{5 / 7}$ core has a map extended to two adjacent planes. For completeness, we have studied the mobility of the a-screw, c-edge and c-screw dislocations and we found that none of them is mobile under resolved shear strains up to 5\%. These results are in accordance with the respective MDD's.

The boundaries of low angle of rotation formed by $\mathrm{C}_{4}$ units are mobile since the $\mathrm{C}_{4}$ units can be identified as glissile a-edge dislocations. These units glide under applied strain perpendicular to the boundary inducing a conservative displacement of the boundary. If a crystal dislocation of the $\mathrm{C}_{4}$ type approaches the boundary an interaction with the dislocations of the boundary is produced that depends on the relative positions and Burgers vectors. In particular, dislocations can either annihilate or be accommodated on the boundary. In turn the boundary can emit dislocations.

For each boundary of $(10 \overline{1} 1),(10 \overline{1} 2)$ and $(10 \overline{1} 3)$ twins, we have considered distinct reference bicrystal structures and we have calculated the corresponding $\gamma$-surfaces to search all possible stable configurations. The core structure of the twin boundaries was found to be dependent on the starting bicrystal structure $\mathrm{P}_{\lambda} / \mathrm{P}_{\mu}$. Although some starting configurations do not present an entirely symmetrical shape, the mirror symmetry was recovered by rigid body displacements parallel to the twin plane and/or during the relaxation process. $4-$, 8- or 5/7-coordianted channels separated by 6-coordinnated channels describe the $(10 \overline{1} 2)$ boundary whereas 6 -coordinated channels uniquely composed the $(10 \overline{1} 3)$ boundary that is the one with lowest energy. The energy calculations show that the low-energy structures only exhibits pure mirror symmetry for $(10 \overline{1} 2)$ and (10 $\overline{1} 3)$ twins.

Acknowledgements: The authors acknowledge the support of the UE (contract HPRN-CT1999-00040) and the Spanish MCYT (BFM2003-08211-C03-03 / FISI). Part of the calculations were performed in CESCA (Centre de Supercomputació de Catalunya). 


\section{References}

[1] A. Béré and A. Serra, Phys. Rev. B 65, 205323 (2002)

[2] A. Béré and A. Serra, Interf. Sci. 9, 149 (2001)

[3] A. Béré and A. Serra, Phys. Rev. B 66, 085330 (2002)

[4] A. Béré and A. Serra, Phys. Rev. B 68, 033305 (2003)

[5] A.N. Efimov, A.O. Lebedv and A.M. Tsaregorodtsev, J.Appl. Crsy. 31, 461 (1998)

[6] L. Liu and J.H. Edgar, Mat. Sci. and Eng. R 37, 61 (2002)

[7] J.E. Northrup, J. Neugebauer and L.t. Romano, Phys. Rev. Lett. 77, 103 (1996).

[8] J. Elsner, R. Jones, P.K. Sitch, V.D. Porezag, M. Elstner, Th. Frauenheim, M.I. Heggie, S. Öberg and P.R. Briddon, Phys. Rev. Lett. 79, 3672 (1997).

[9] A.F. Wright and U. Grossner, Appl. Phys. Lett. 73, 2751 (1998).

[10] J.E. Northrup, Appl. Phys. Lett. 78, 2288 (2001).

[11] Z.Z. Bandic, T.C. McGill and Z. Ikonic, Phys. Rev. B 56, 3564 (1997)

[12] V. Potin, P. Ruterana, G. Nouet, R.C. Pond and H. Morkoç, Phys. Rev. B 61, 5587 (2000).

[13] V. Narayanan, K. Lorenz, W. Kim and S. Mahajan, Philos. Mag. A 82, 885 (2002).

[14] P. Vennéguès, B. Beaumont, V. Bousquet, M. Vaille and P. Gibart, J. Appl. Phys. 87,4175 (2000).

[15] F.A. Ponce MRS bull. 22, 51 (1997)

[16] J. Amon, F. Dumke and G. Müller, J. Crystal Growth 187, 1 (1998)

[17] M.H.Yoo: Metall Trans.B 1981, 12A, 409

[18] A. Serra and B.J. Bacon, Acta metall. Mater. 43, 4465 (1995).

[19] A.Serra and D.J. Bacon, Phil. Mag. A 73, 333 (1996)

[20] A. Serra, D.J. Bacon, and R.C. Pond, Acta Mater. 47, 1425 (1999).

[21] R.C. Pond, A. Serra, and D.J. Bacon, Acta Mater. 47, 1441 (1999).

[22] L. Cheng, Z. Zhang, G. Zhang and D. Yu, Appl. Phys. Lett., 71, 3694 (1997)

[23] J.P Hirth and J. Lothe, Theory of Dislocations, John Wiley, New York (1982).

[24] R.C.Pond: in Dislocation in Solids, F.R.N.Nabarro, ed., North Holland Publishing Co., Amsterdam, 8, 1 (1989)

[25] R.C. Pond and W Bollman, Phil. Trans. R. Soc. A292, 449, (1979)

[26] R.C.Pond, Proc. R. Soc. London A 357, 471 (1977)

[27] V. Vitek, A.P. Sutton, D.A. Smith and R.C. Pond: in Grain Boundary Structure and Kinetics, R.W. Balluffi, ed., ASM, Metals Park, OH, 115 (1980)

[28] R.C.Pond, D.J. Bacon, A.Serra and A.P. Sutton, Metallurgical Transactions A, 22, 1185 (1991)

[29] P.C. Gehlen, J.R. Beeler and R.I. Jafee (Eds.) Interatomic Potentials and Simulation of Lattice Defects, Battelle Institute Materials Science Coll., Plenum Press, (1972).

[30] K. Kim, W.R.L. Lambrech and B. Segall, Mat. Res. Soc. Symp. Proc. 395, 399 (1996)

[31] A. Béré, P. Vermaut, A. Hairie, E. Paumier, P. Ruterana and G. Nouet, Comp. Mat. Sci. 294/296, 223 (1999)

[32] N. Aïchoune, V. Potin, P. Ruterana, A. Hairie, G. Nouet and E. Paumier, Comp. Mat. Sci. 17, 380 (2000).

[33] W.H. Moon and H.J. Hwang, Physics Letters A 315, 319 (2003)

[34] J. Nord, K. Albe, P. Erhart and K. Nordlund, J. Phys.: Cond.Matter, 15, 5649 (2003)

[35] P.N. Keating, Phys. Rev. 145, 637 (1966)

[36] F.H. Stillinger and T.A. Weber, Phys. Rev. B 38, 1537 (1985).

[37] M. Ichimura, Phys. Stat. Sol. (a) 153, 431 (1996).

[38] P. Ruterana, A. Béré, B. Barbaray, G. Nouet, A. Hairie, A. Paumier, A. Salvabor, A. Botchkarev and H. Morkoç, Phys. Rev. B. 59, 15917 (1999) 
[39] D.B. Holt, J.Phys. Chem. Solids 30, 1297, (1969).

[40] J.E. Northrup, J. Neugebauer and L.t. Romano, Phys. Rev. Lett. 77, 103 (1996).

[41] V. Potin, G. Nouet and P. Ruterana, Appl. Phys. Lett. 74, 947 (1999).

[42] B. Lebouvier, A. Hairie, F. Hairie, G. Nouet and E. Paumier, Mat. Sci. Forum 207209, 277 (1996).

[43] D. Kincaid and W. Cheney, Numerical analysis: Mathematics of scientific computing. Brooks/Cole Publishing company, USA (1991)

[44] V.A. Savastenko and A.U. Shelog, Phys. Status Solidi A, 48, K135 (1978).

[45] V. Vitek, R.C. Perrin and D.K. Bowen, Phil. Mag. 21, 1049 (1970).

[46] Y. Xin, S. J. Pennycook, N.D. Browning, P.D. Nellist, S. Sivanathan, F. Ommnès, B. Neaumont, J.P. Faurie and P. Gibart, Appl. Phys. Lett. 72, 2680, (1998).

[47] W. Qian, G.S. Rohrer, M. Skoeronski, K. Doveerspike, L.B. Rowland and D.K. Gaskill, Appl. Phys. Lett. 67, 2284 (1995).

[48] P. Vennéguès, B. Beaumont, M. Vaille and P. Gibart, Phys. Rev. Lett. 70, 2434 (1997).

[49] S.M. Lee, M.A. Belkhir, X.Y. Zhu, Y.H. Lee, Y.G. Hwang, Th. Frauenheim, Phys. Rev. B 61, 16033 (2000).

[50] J. Chen, P. Ruterana and G. Nouet, Phys. Rev.B. 67, 205210 (2003)

[51] A.N. Kiselev, F. Sarrazit, E.A. Stepantsov, E. Olsson, T. Claeson, V.I. Bondarenko, R.C.Pond and N.A. Kieselev; Phil Mag. A, 76, 633 (1997) 


\section{Table captions}

TABLE 1: Parameters for the Stillinger-Weber potential and calculated structural properties of wurtzite structure. Experimental values are in parentheses.

TABLE 2: The shear modulus $\mu$ as a function of the elastic constants $C_{i j}$ and the theoretical pre-logarithmic factors $\left(\mathrm{A}_{\mathrm{th}}^{\mathrm{S}-\mathrm{W}}\right.$ and $\left.\mathrm{A}_{\mathrm{th}}^{\mathrm{Exp}}\right)$ for the edge and screw dislocations. The $\mathrm{S}-\mathrm{W}$ and experimental elastic constants are reported in Ref. 32.

TABLE 3: Core radius $\left(r_{c}\right)$, core energy $\left(E_{c}\right)$, slope $\left(A_{0}\right)$ and total energy obtained with the S-W potential $\left(E_{T}^{S W}\right)$ for dislocation structures. The total energy $E_{T}^{F P}$ given by firstprinciples calculations is included for comparison.

Table 4. Data for [0001] tilt boundaries. The angle $\theta$ refers to the misorientation angle and $\Delta \mathrm{E}$ is the lower structure energy. The sequence of environments for one period along the boundary plane is given for the structure of lower energy. 
$\underline{\text { Figure captions }}$

Fig. 1: (a) Periodic images along the $\mathrm{Z}$ direction of an interface. (b) Dichromatic complex of $\Sigma 7$ showing an antimirror plane. On the left the studied interface $\mathrm{A}($ white)/B(black); on the right the boundary due to the periodic conditions $\mathrm{C}($ black)/D(white). The closed circles represent $\mathrm{N}$ atoms whereas the open circles represent $\mathrm{Ga}$.

Fig.2: Two-body terms as a function of each interaction type. The 1 and 2 digits refer to parameters given in Ref.[32] and the present work respectively.

Fig. 3: Relaxed configuration of the $\vec{a}$ edge dislocation when the origin of displacements is located between two closely spaced $(10 \overline{1} 0)$ planes: (a) Core described by 5/7-coordinated channels. It presents wrong bond $(\mathrm{Ga}-\mathrm{Ga})$ in this plane and $(\mathrm{N}-\mathrm{N})$ on the planes above and below. (b) Map of differential displacements of neighbouring pairs of atoms.

Fig. 4: Relaxed configuration of the $\vec{a}$ edge dislocation when the origin of displacements is located between two widely spaced $(10 \overline{1} 0)$ planes: (a) Core described by 4-coordinated channels. It is characterized by four bonds practically in the same plane. (b) Map of differential displacements of neighbouring pairs of atoms.

Fig. 5: Relaxed configuration of the $\vec{a}$ edge dislocation when the origin of displacements is located between two widely spaced (10 $\overline{1} 0$ ) planes (more precisely in the hexagon centre): (a) Core described by 8-coordinated channels. The core has a dangling bond. (b) Map of differential displacements of neighbouring pairs of atoms.

Fig.6: Total energy stored in a cylinder of radius $R$ as a function of $\ln (R)$ for the $\vec{a}$ dislocations.

Fig. 7: Map of differential displacements of neighbouring pairs of atoms of the relaxed core configurations of the $\vec{a}$ screw dislocation. The origin of displacements is located between (a) two closely spaced and (b) two widely spaced (10 $\overline{1} 0)$ planes. 
Fig.8: (a) Core configurations and (b) map of differential displacements of neighbouring pairs of atoms of the $\vec{c}$ edge dislocation with origin of displacements located between two closely spaced (10 $\overline{1} 0)$ planes.

Fig.9: (a) Core configurations and (b) map of differential displacements of neighbouring pairs of atoms of the $\vec{c}$ edge dislocation with origin of displacements located between two widely spaced (10 $\overline{1} 0)$ planes.

Fig. 10: Map of differential displacements of neighbouring pairs of atoms of the relaxed configurations of the $\vec{c}$ screw dislocation. (a) The origin of displacements is in a plane of mirror glide symmetry. (b) The origin of displacements coincides with the three-fold axis

Fig.11: Atomic structures of the $\Sigma 19\left(\theta=13.4^{\circ}\right)(3 \overline{5} 20)$ tilt boundary. The structures in (a, unrelaxed) and (b, relaxed) are obtained from a starting configuration formed by the $a(\lambda) / b(\mu)$ cnid whereas the structure in $(c)$ is obtained from $b(\lambda) / b(\mu)$. The structures in $(d)$ and (e) correspond to translation states of the $a(\lambda) / b(\mu)$ starting configuration. The structure in (b) is the most favourable energetically.

Fig.12: Atomic structures of the $\Sigma 7\left(\theta=21.6^{\circ}\right)(2 \overline{3} 10)$ tilt boundary. The structure in (a) is obtained from a starting configuration formed by the $a(\lambda) / b(\mu)$ cnid whereas the structure in (b) is obtained from $b(\lambda) / b(\mu)$.The structure in (a) is the most favourable energetically.

Fig.13: Atomic structure of lower energy of the $\Sigma 43\left(\theta=44.8^{\circ}\right)(1 \overline{7} 60)$ tilt boundary.

Fig.14: Atomic structure of lower energy of the $\Sigma 49\left(\theta=16.3^{\circ}\right)(3 \overline{8} 50)$ tilt boundary.

Fig.15: Atomic structures of the $\Sigma 13\left(\theta=27.8^{\circ}\right)$ (2750) tilt boundary. (a) Structure formed by three n-coordinated channels. (b) Structure of lower energy formed by three 5/7-channels of alternate orientations. 
Fig.16: Atomic structures of the $\Sigma 13\left(\theta=32.2^{\circ}\right)(1 \overline{4} 30)$ tilt boundary. (a) Structure of lower energy formed by two 5/7-channels of alternate orientations. (b) Structure formed by one 5/7 and one 4-coordinated channels.

Fig.17: Mobility of tilt boundary under a perpendicular applied shear strain perpendicular to the boundary plane. a) reference boundary. b) and c) movement of the $\mathrm{C} 4$ cores under a strain $\varepsilon_{\mathrm{xy}}= \pm 0.04$.

Fig.18: Stable positions of edge dislocation $\left(\mathrm{C}_{4}\right)$ located near the boundary. a) The dislocation has the same glide plane and sign than the $\mathrm{C}_{4}$ unit in the boundary. The repulsion pushed the $\mathrm{C}_{4}$ unit out of the boundary. b) The dislocation is between two $\mathrm{C}_{4}$ units. It is in equilibrium under the short ranged stress field created by the boundary. c) The dislocation has the same glide plane but opposite sign than the $\mathrm{C}_{4}$ unit in the boundary. The restoration of the perfect crystal confirms the dislocation character of the structural units.

Fig.19: Same configuration as in Fig.18b under an applied strain. b) The dislocation has been incorporated to the boundary. c) When the strain is reversed the boundary approaches the dislocation that is incorporated to the boundary. Notice that the lower $\mathrm{C}_{4}$ has converted into a $\mathrm{C}_{8}$.

Fig.20: Lowest energy structure of the $(10 \overline{1} 1)$ twin projected along [11 $\overline{2} 0]$. The four symbols represent the four layers in the stacking sequence. Hexagonal unit cells are shown in outline. Squares correspond to A-type layers, triangle to a-type, circles to B-type and crosses to b-type layers in the stacking sequence of the wurtzite structure along [0001].

Fig.21: Atomic structures of the (10 $\overline{1} 2)$ twin projected along [11 $\overline{2} 0]$. The structure in a) exhibits a four-coordinated channel (4-interface), in b) an eight-coordinated channel (8interface) and in c) a five- and seven-coordinated channels (5/7-interface).

Fig.22: Lowest energy structure of the (10 $\overline{1} 3)$ twin projected along [11 $\overline{2} 0]$. 
TABLE I

\begin{tabular}{|c|c|c|c|c|c|c|}
\hline & \multicolumn{3}{|c|}{ Aïchoune et al. $^{20}$} & \multicolumn{3}{|c|}{ Present work } \\
\hline & Ga-N & $\mathrm{Ga}-\mathrm{Ga}$ & $\mathrm{N}-\mathrm{N}$ & Ga-N & $\mathrm{Ga}-\mathrm{Ga}$ & $\mathrm{N}-\mathrm{N}$ \\
\hline \multicolumn{7}{|c|}{ Parameters of the Stillinger-Weber potential } \\
\hline$\varepsilon(\mathrm{eV} / \mathrm{bond})$ & 2.17 & 0.655 & 0.655 & 2.17 & 1.2 & 1.2 \\
\hline$\sigma(\stackrel{0}{A})$ & 1.695 & 2.038 & 1.302 & 1.695 & 2.1 & 1.3 \\
\hline$\lambda$ & 32.5 & 26.76 & 26.76 & 32.5 & 32.5 & 32.5 \\
\hline $\mathrm{a}\left(\mathrm{r}_{\mathrm{c}} / \sigma\right)$ & 1.8 & 1.8 & 1.8 & 1.8 & 1.6 & 1.8 \\
\hline A & 7.917 & 7.917 & 7.917 & 7.917 & 7.917 & 7.917 \\
\hline B & 0.720 & 0.720 & 0.720 & 0.720 & 0.720 & 0.720 \\
\hline \multicolumn{7}{|c|}{ Lattice parameters } \\
\hline $\mathrm{a}(\stackrel{0}{\mathrm{~A}})$ & \multicolumn{3}{|c|}{$3.23(3.19)^{\mathrm{a}}$} & \multicolumn{3}{|c|}{$3.19(3.19)^{\mathrm{a}}$} \\
\hline$c(\stackrel{0}{A})$ & \multicolumn{3}{|c|}{$5.28(5.18)^{\mathrm{a}}$} & \multicolumn{3}{|c|}{$5.20(5.18)^{\mathrm{a}}$} \\
\hline \multicolumn{7}{|c|}{ Inversion domain boundaries excess energies $\Delta \mathrm{E}$} \\
\hline \multirow[t]{2}{*}{$\Delta \mathrm{E}\left(\mathrm{mJ} / \mathrm{m}^{2}\right)$} & \multicolumn{3}{|c|}{ Not stable } & \multicolumn{3}{|c|}{$2590(2663)^{b}$} \\
\hline & \multicolumn{3}{|c|}{$702(400)^{b}$} & \multicolumn{3}{|c|}{$949(400)^{b}$} \\
\hline
\end{tabular}

${ }^{a}$ Experimental data.

${ }^{b}$ Ref. 7 
TABLE II

\begin{tabular}{cccc}
\hline \hline Defect & $\mu$ & $\mathrm{A}_{\text {th }}^{\text {S-W }}$ & $\mathrm{A}_{\text {th }}^{\text {Exp }}$ \\
\hline Prism edge $(\overrightarrow{\mathrm{a}})$ & $(1-v) \frac{\mathrm{C}_{11}^{2}-\mathrm{C}_{12}^{2}}{2 \mathrm{C}_{11}}$ & 0.77 & 0.86 \\
Basal screw $(\overrightarrow{\mathrm{a}})$ & $\left(\mathrm{C}_{44} \mathrm{C}_{66}\right)^{1 / 2}$ & 0.56 & 0.58 \\
Basal edge $(\overrightarrow{\mathrm{c}})$ & $\mu_{\mathrm{B}}^{\mathrm{e}}$ & 2.16 & 2.24 \\
Prism screw $(\overrightarrow{\mathrm{c}})$ & $\mathrm{C}_{44}$ & 1.46 & 1.40 \\
\hline
\end{tabular}

$$
\mu_{B}^{\mathrm{e}}=(1-v)\left(\sqrt{\mathrm{C}_{11} \mathrm{C}_{33}}+\mathrm{C}_{13}\right)\left(\frac{\mathrm{C}_{44}\left(\sqrt{\mathrm{C}_{11} \mathrm{C}_{33}}-\mathrm{C}_{13}\right.}{\mathrm{C}_{11}\left(\sqrt{\mathrm{C}_{11} \mathrm{C}_{33}}+\mathrm{C}_{13}+2 \mathrm{C}_{44}\right)}\right)^{1 / 2}
$$


TABLE III:

\begin{tabular}{cccccccc}
\hline \hline Defect & $\begin{array}{c}\text { Type } \\
\text { Core }\end{array}$ & $\begin{array}{c}\mathrm{r}_{\mathrm{c}} \\
(\AA)\end{array}$ & $\begin{array}{c}\mathrm{E}_{\mathrm{c}} \\
(\mathrm{eV} / \AA)\end{array}$ & $\begin{array}{c}\mathrm{A}_{0} \\
(\mathrm{eV} / \AA)\end{array}$ & $\begin{array}{c}\mathrm{E}_{\mathrm{T}}^{\mathrm{SW}}(\mathrm{eV} / \AA) \\
(\mathrm{R}=20 \AA)\end{array}$ & $\begin{array}{c}\mathrm{E}_{\mathrm{T}}^{\mathrm{SW}} \\
(\mathrm{eV} / \AA)\end{array}$ & $\begin{array}{c}\mathrm{E}_{\mathrm{T}}^{\mathrm{FP}} \\
(\mathrm{eV} / \AA)\end{array}$ \\
\hline Prism edge $(\overrightarrow{\mathrm{a}})$ & $5 / 7$ & 6.7 & 1.46 & 0.79 & 2.38 & & \\
& 8 & 6.7 & 1.72 & 0.78 & 2.57 & & \\
& 6.7 & 1.72 & 0.78 & 2.57 & $\begin{array}{c}1.95^{\mathrm{a}} \\
(\mathrm{R}=8.5 \AA)\end{array}$ & $2.19^{8}(\mathrm{R}=8.5 \AA)$ \\
Basal screw ( $\overrightarrow{\mathrm{a}})$ & $\mathrm{R} 2$ & 6.7 & 1.20 & 0.59 & 1.80 & & \\
Basal edge $(\overrightarrow{\mathrm{c}})$ & $\mathrm{S}_{2}$ & 8.5 & 3.68 & 1.99 & 5.55 & & \\
Prism screw $(\overrightarrow{\mathrm{c}})$ & $\mathrm{T}_{3}$ & 6.7 & 2.82 & 1.35 & 4.3 & $3.30^{\mathrm{a}}$ & $4.00^{10}(\mathrm{R}=7.5 \AA)$ \\
& & & & & & $(\mathrm{R}=7.5 \AA)$ & $4.88^{8}(\mathrm{R}=8.5 \AA)$ \\
\hline \hline
\end{tabular}

${ }^{\mathrm{a}}$ The total relaxable region is constrained to the cylinder of radius $\mathrm{R}$ given in parentheses. 
TABLE IV

\begin{tabular}{ccccc}
\hline \hline$\Sigma$ & Indices & $\theta$ & Structure $^{*}$ & $\Delta \mathrm{E}\left(\mathrm{mJ} / \mathrm{m}^{2}\right)$ \\
\hline 37 & $(3 \overline{7} 40)$ & 9.3 & $6_{2} 6_{1} 6_{2} 6_{1} 6_{2} 75$ & 1286 \\
19 & $(2 \overline{5} 30)$ & 13.4 & $6_{2} 6_{1} 6_{2} 75$ & 1518 \\
49 & $(3 \overline{8} 50)$ & 16.3 & $6_{2} 6_{1} 6_{2} 756_{2} 6_{1} 4$ & 1692 \\
7 & $(1 \overline{3} 20)$ & 21.6 & $6_{2} 75$ & 1827 \\
13 & $(2 \overline{7} 50)$ & 27.8 & $6_{3} 757575$ & 2185 \\
13 & $(1 \overline{4} 30)$ & 32.2 & 7575 & 1528 \\
43 & $(1 \overline{7} 60)$ & 44.8 & $6_{3} 756_{3} 6_{3} 75$ & 1834 \\
\hline \hline
\end{tabular}

$(*)$ : A $6_{1}$ unit is crossed by the interface plane (see fig. $11 \mathrm{~b}$ ) whereas it passes symmetrically between two $6_{2}$. A $6_{3}$ unit is related to corrugated interface (see fig.11d). 
On the atomic structures, mobility and interactions of extended defects in GaN: dislocations, tilt and twin boundaries

Antoine Béré and Anna Serra
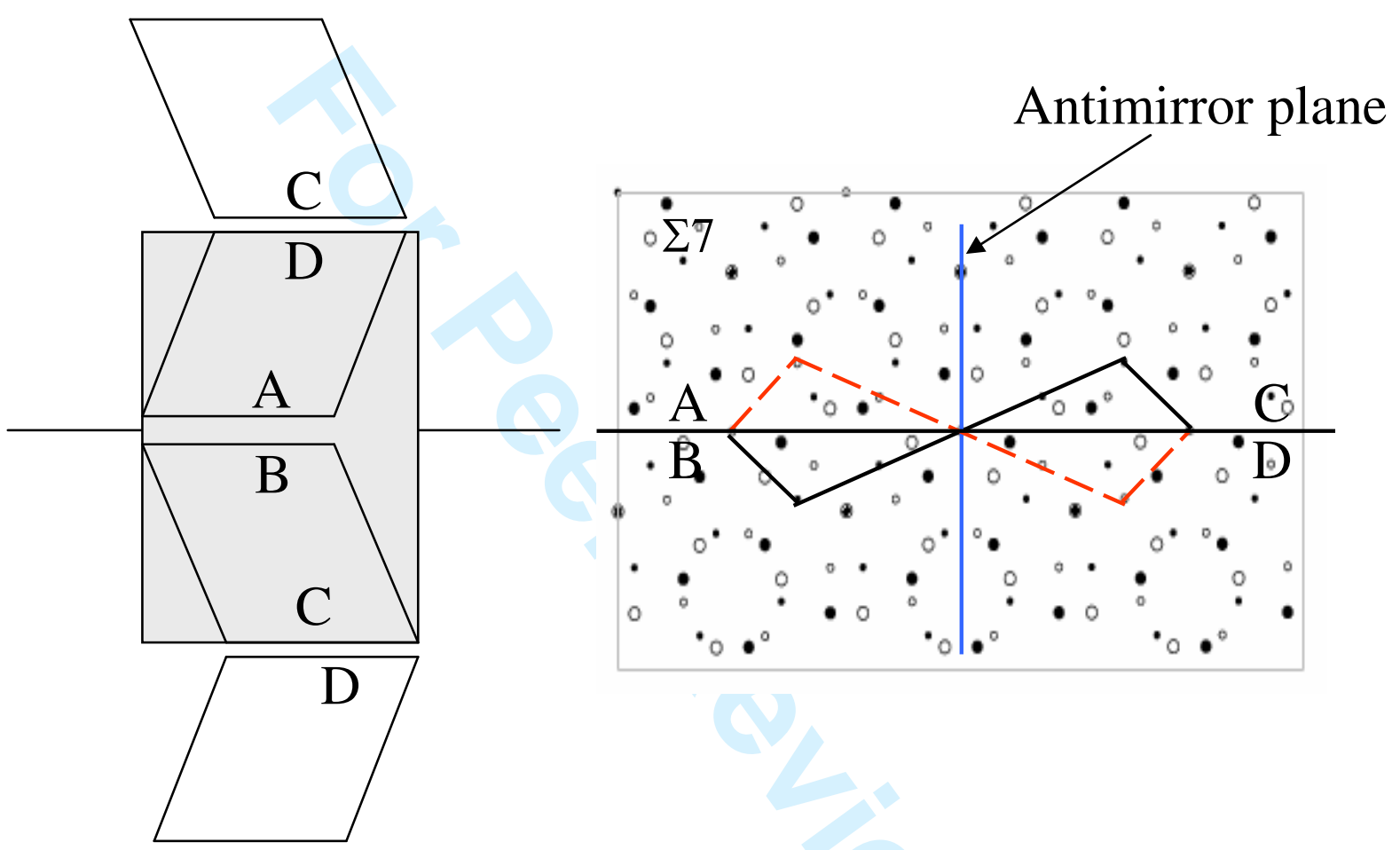

a)

b)

Fig.1 


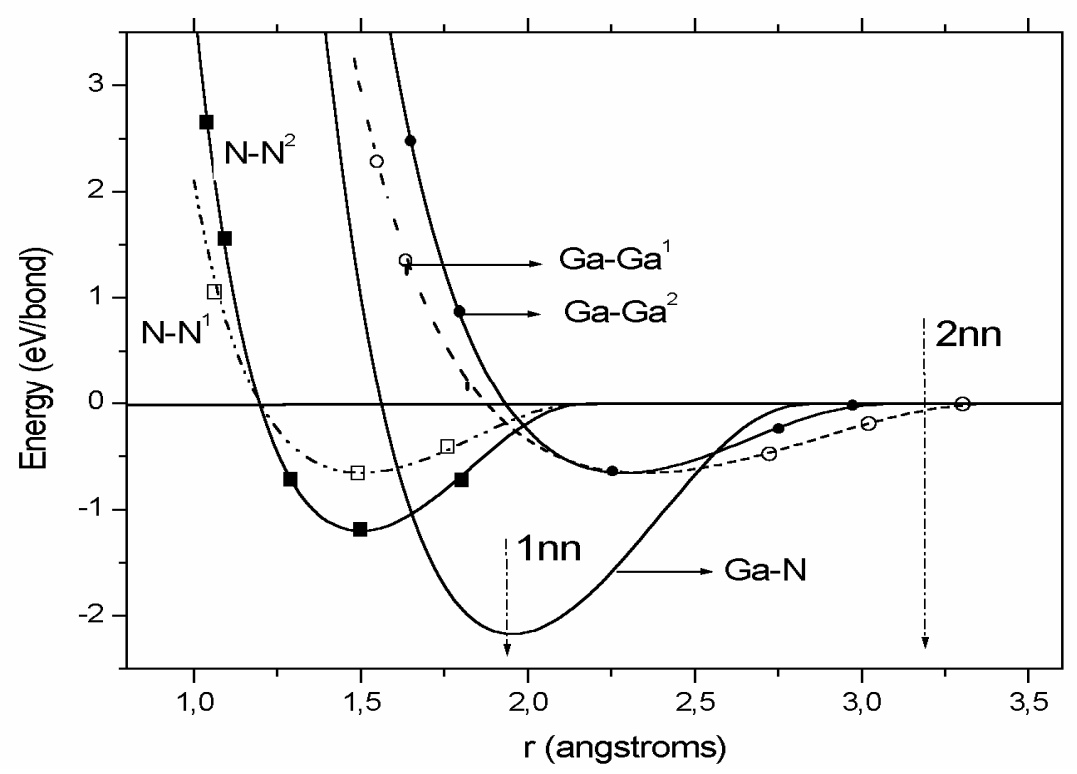

Fig.2 
a)

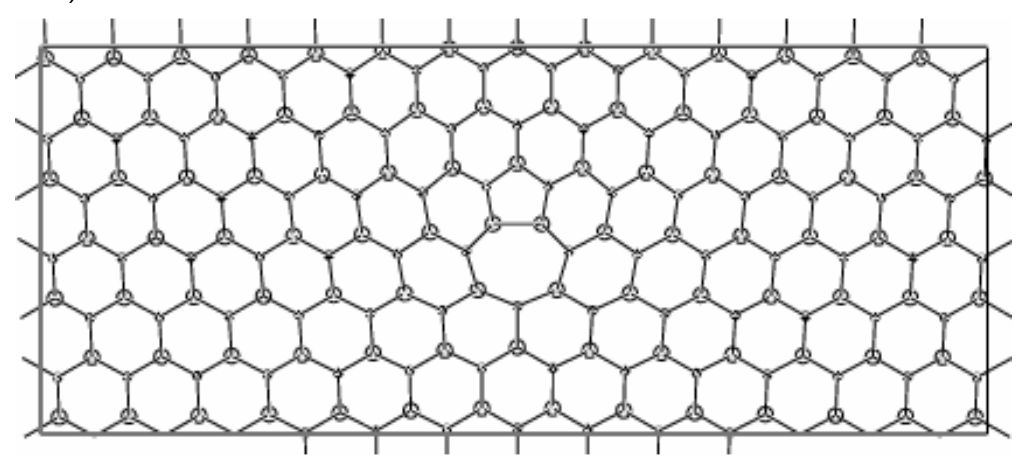

b)

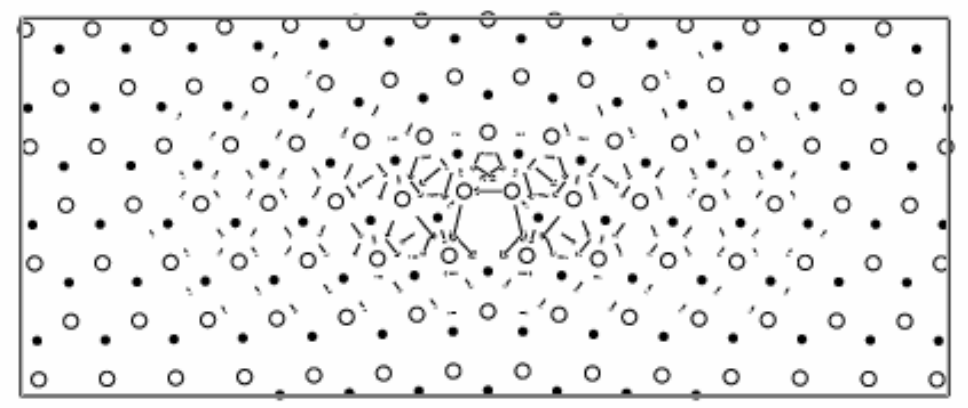

Fig.3 
a)

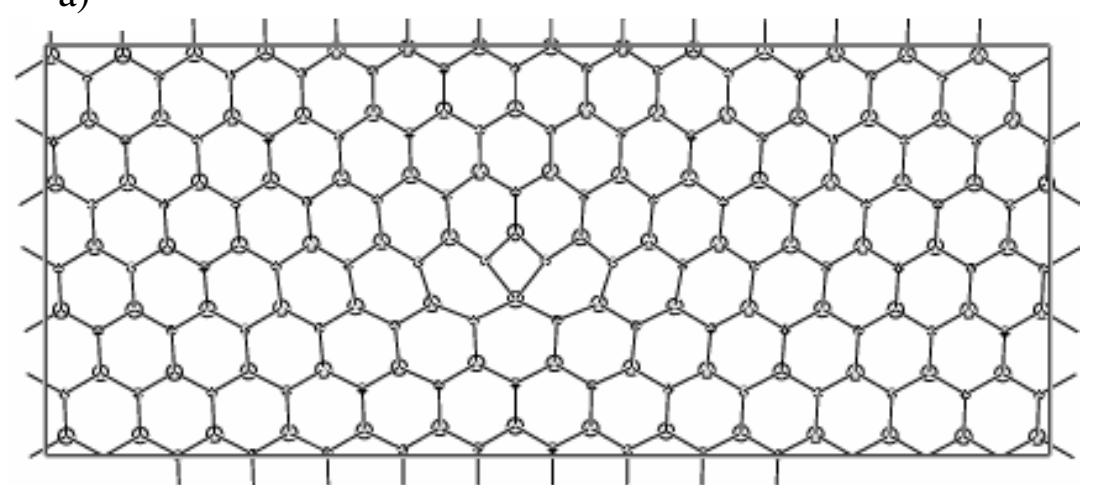

b)

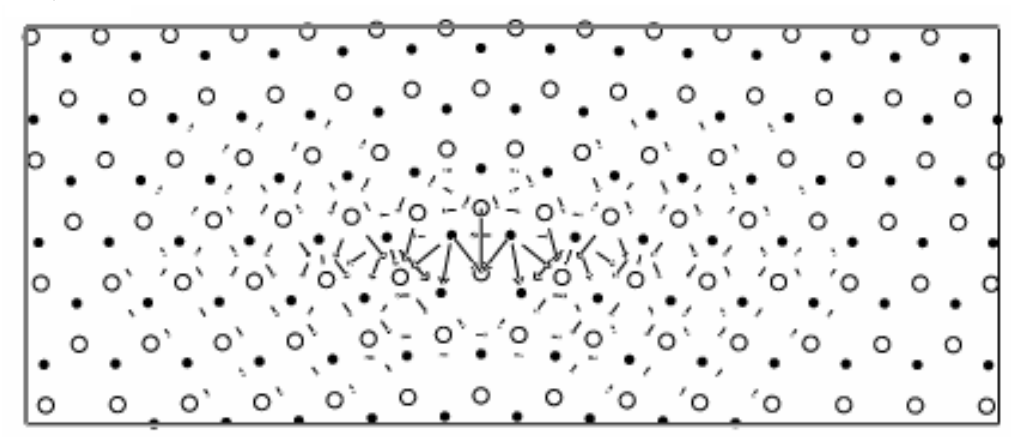

Fig. 4 
a)

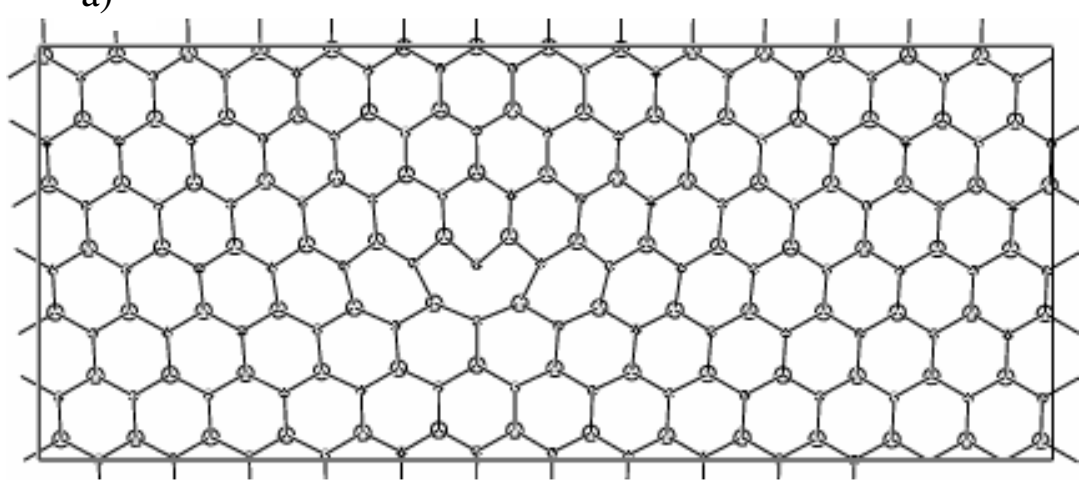

b)

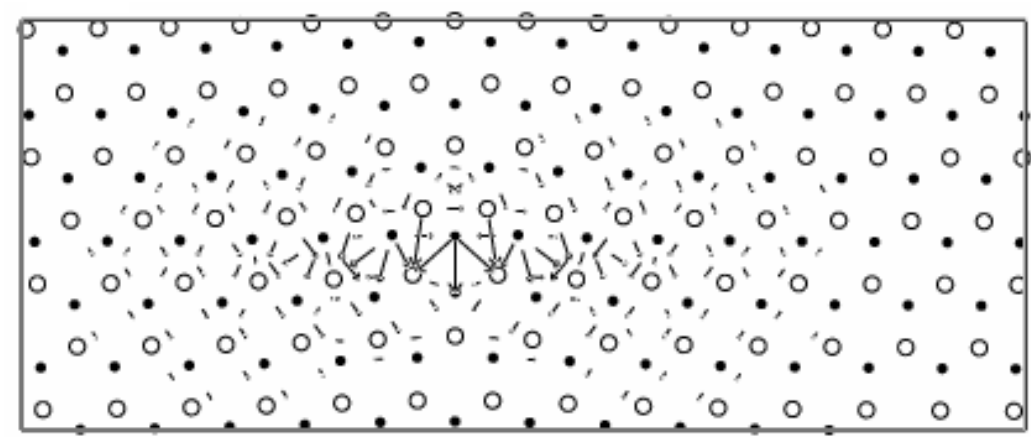

Fig. 5 


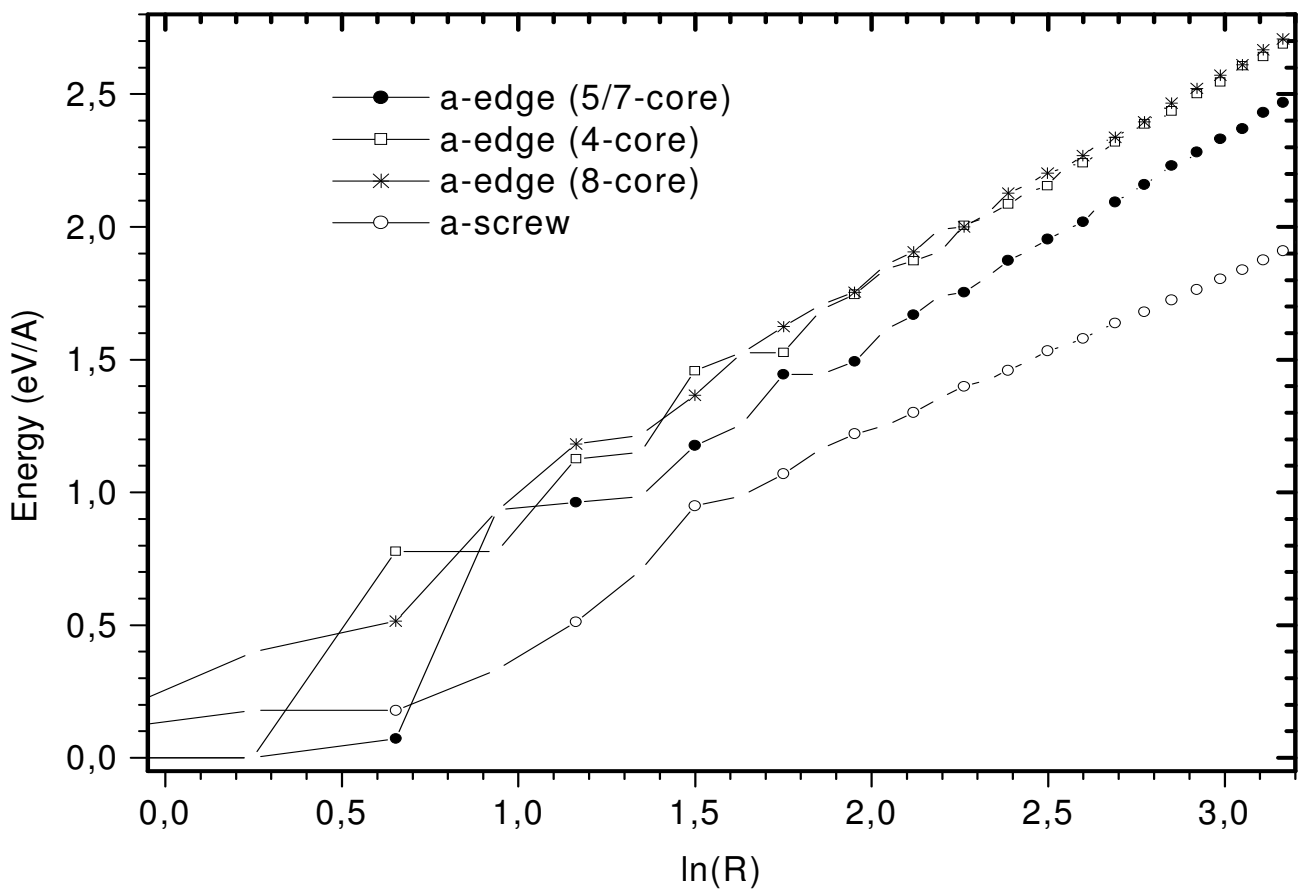

Fig.6 
a)

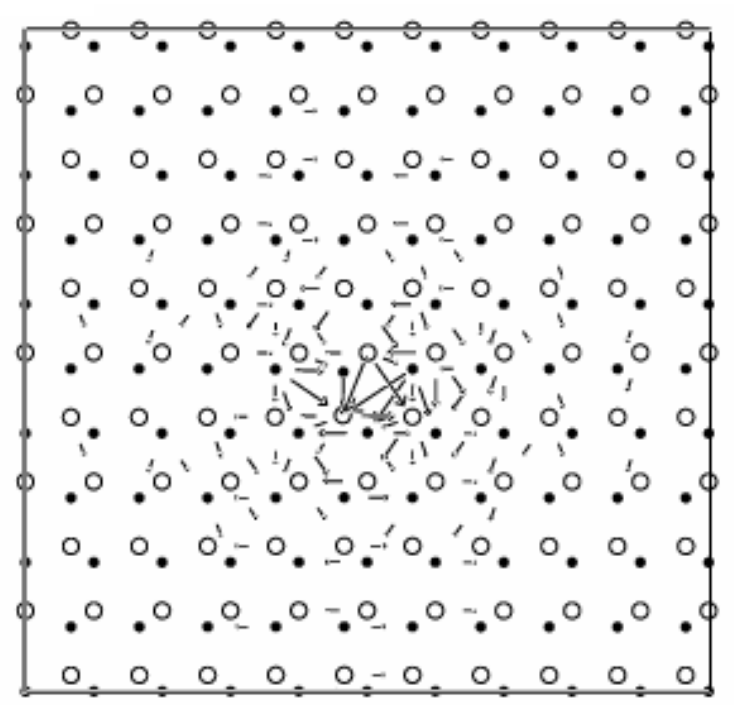

b)



Fig. 7 

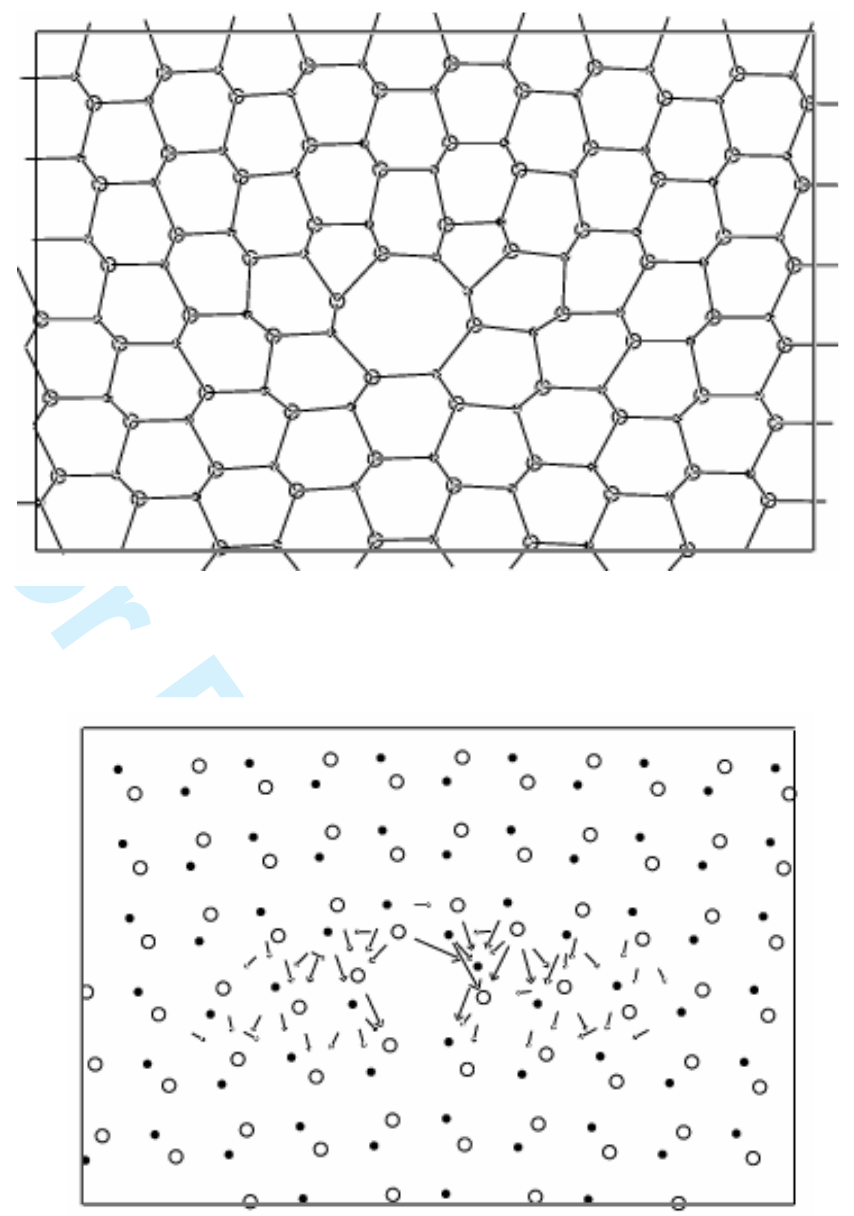

Fig. 8 


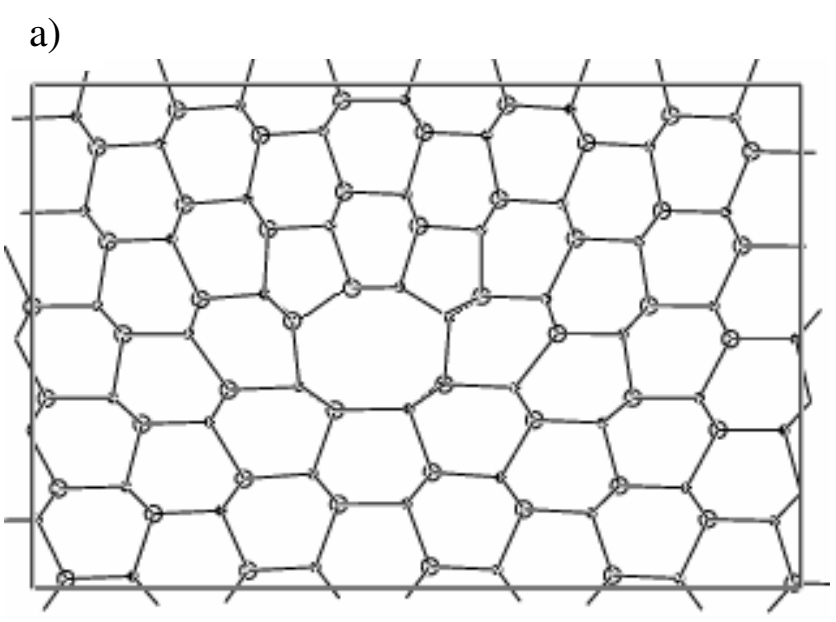

b)

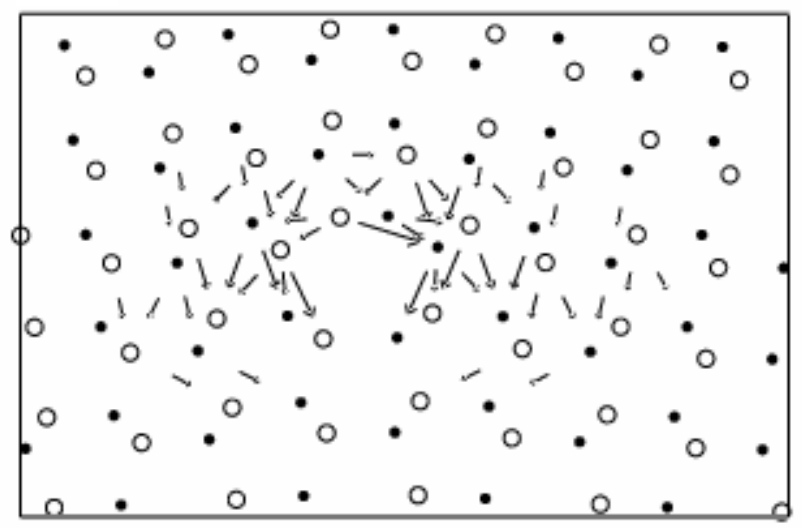

Fig.9 
a)

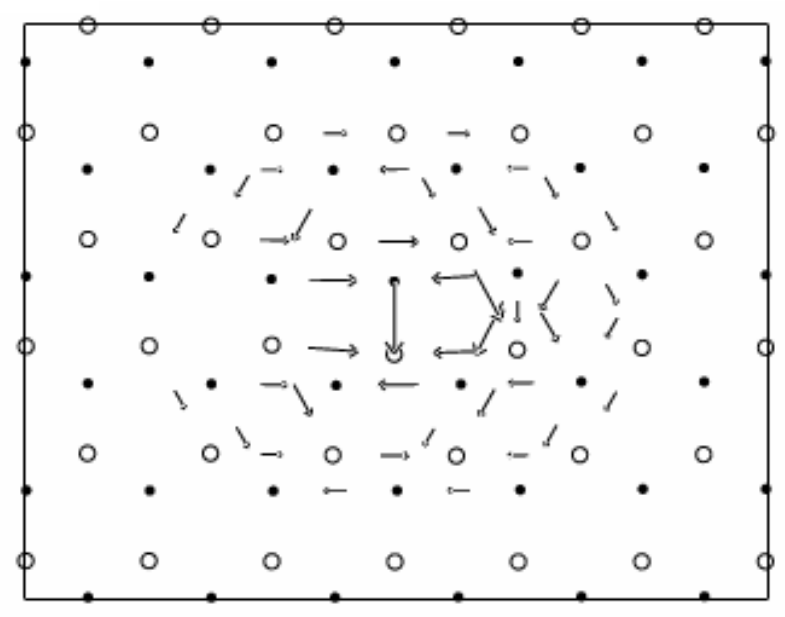

b)

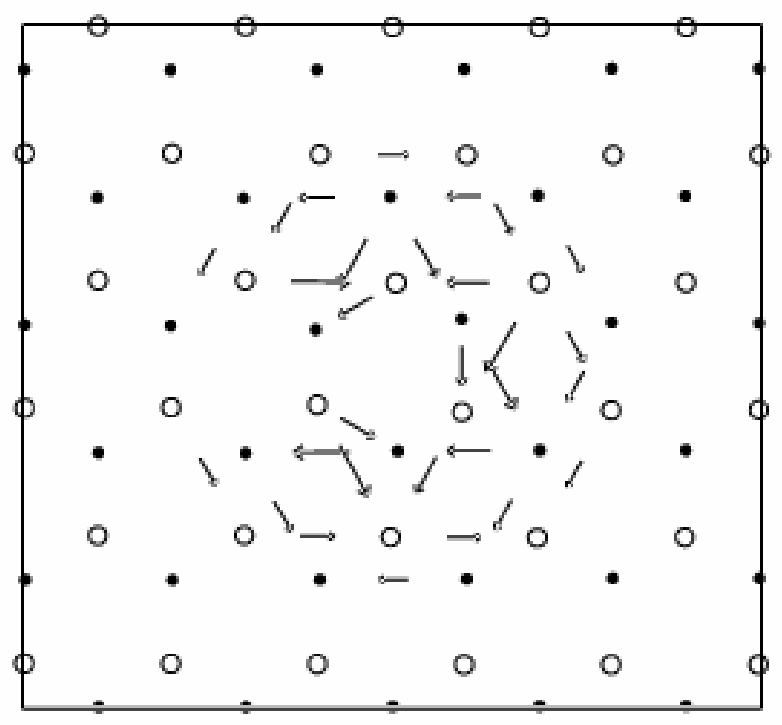

Fig. 10 


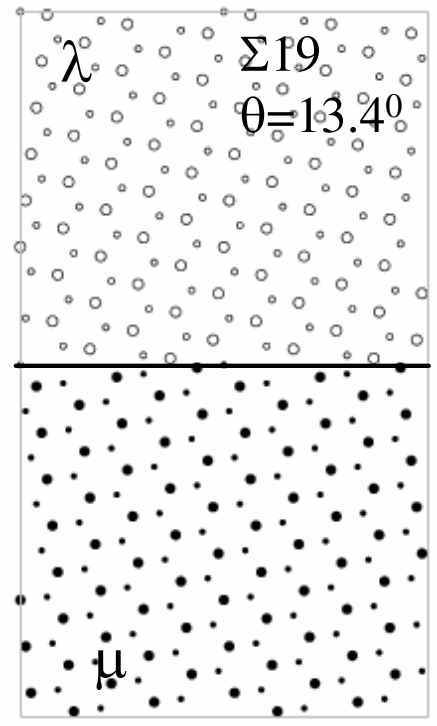

a)

b)

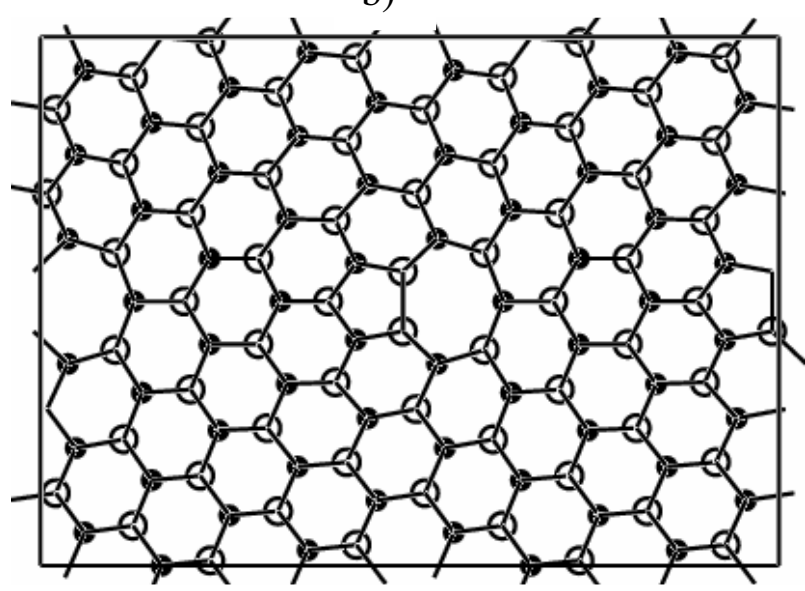

d)

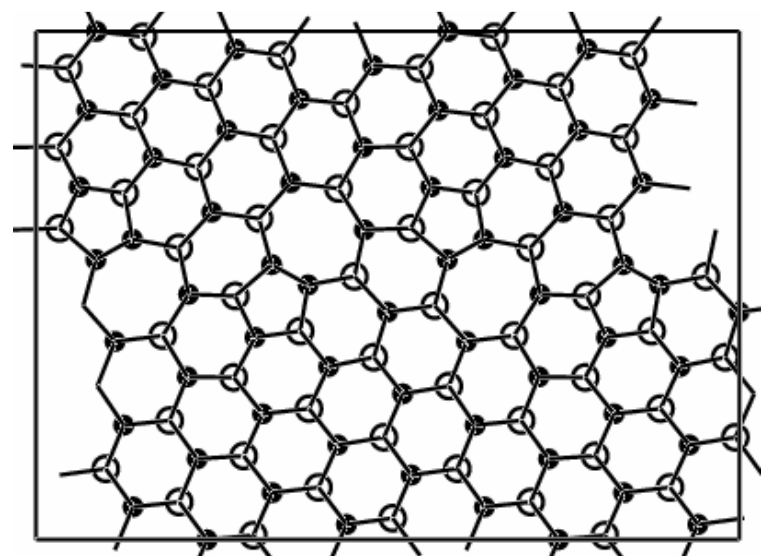

c)



e)

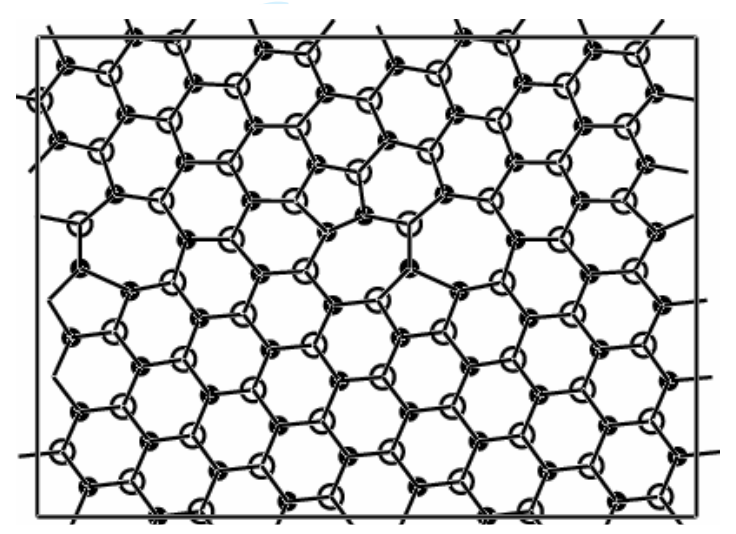

Fig.11 
a)

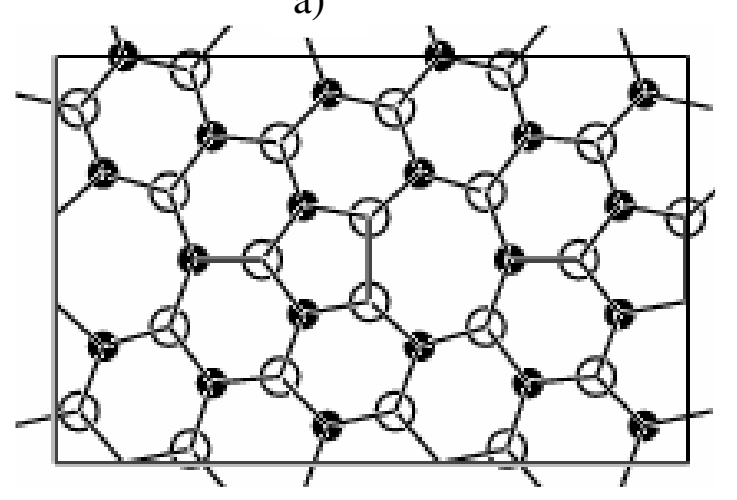

b)

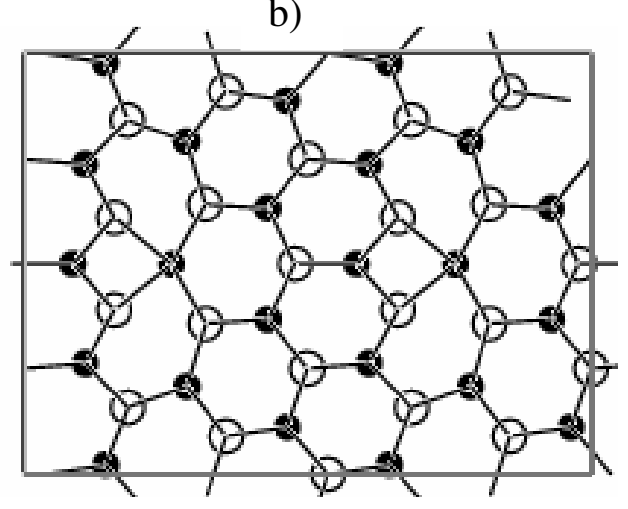

Fig. 12 


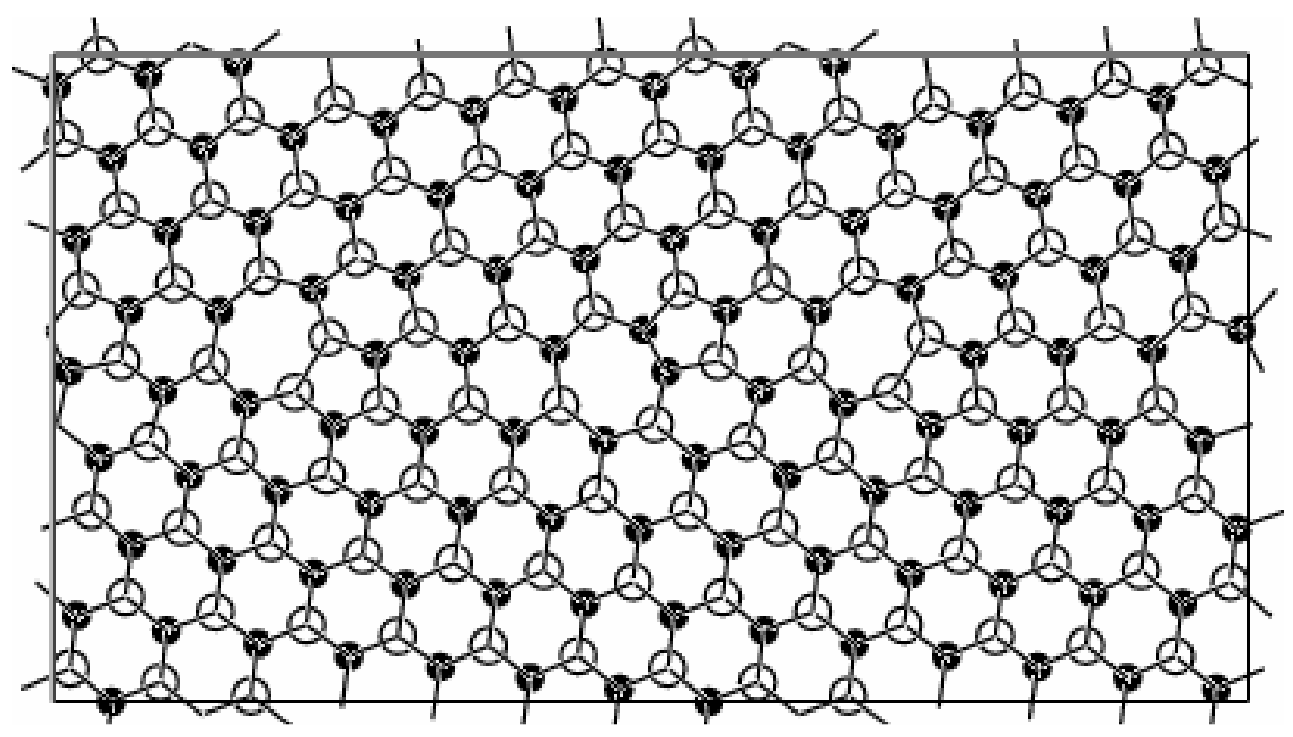

Fig.13 ( 543$)$ 


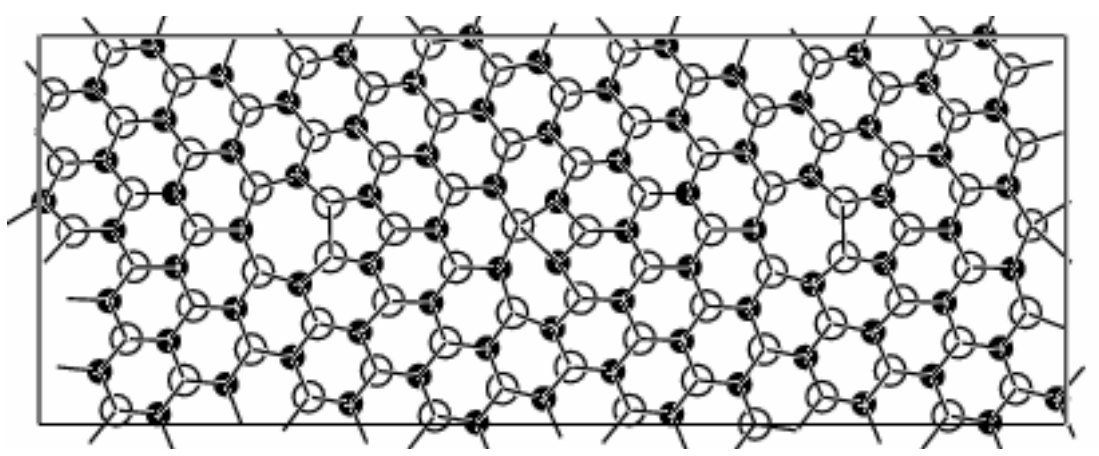

Fig.14 ( 249$)$ 
a)

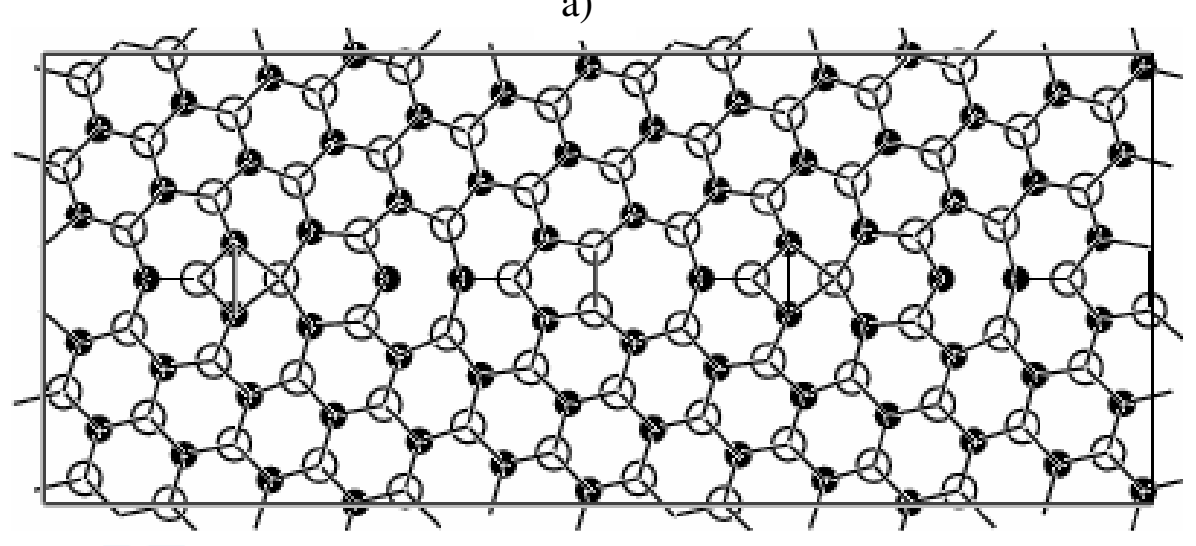

b)

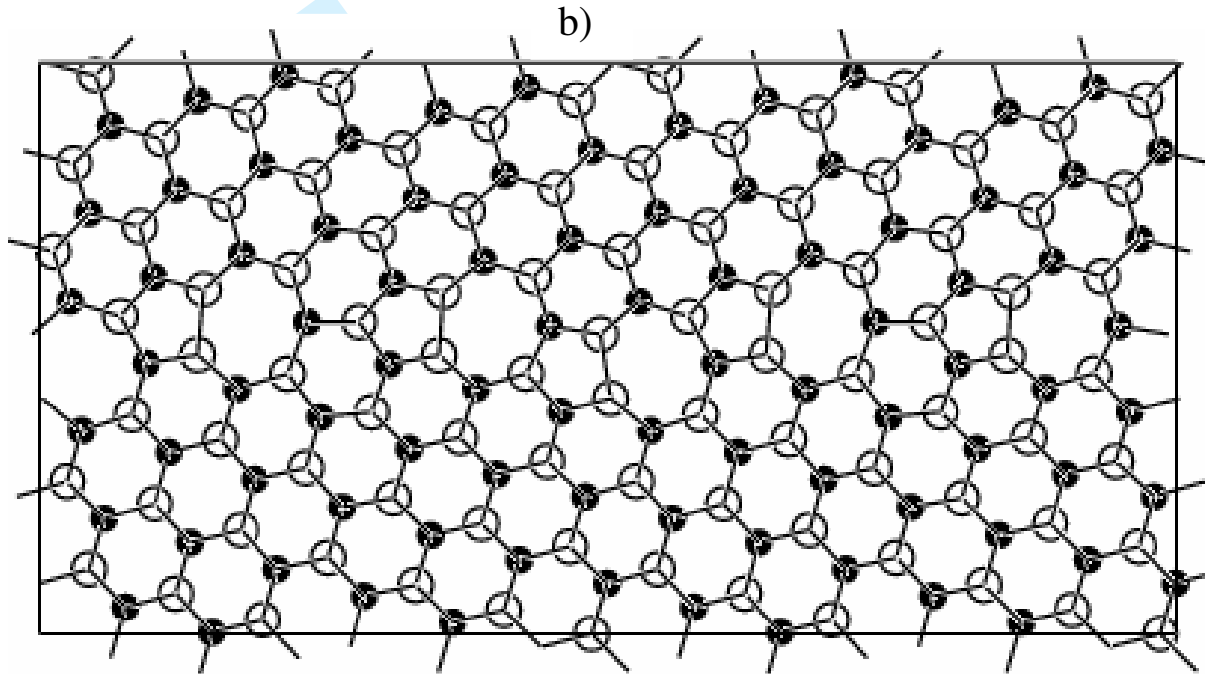

Fig.15 ( $\Sigma 13 b)$ 
a)
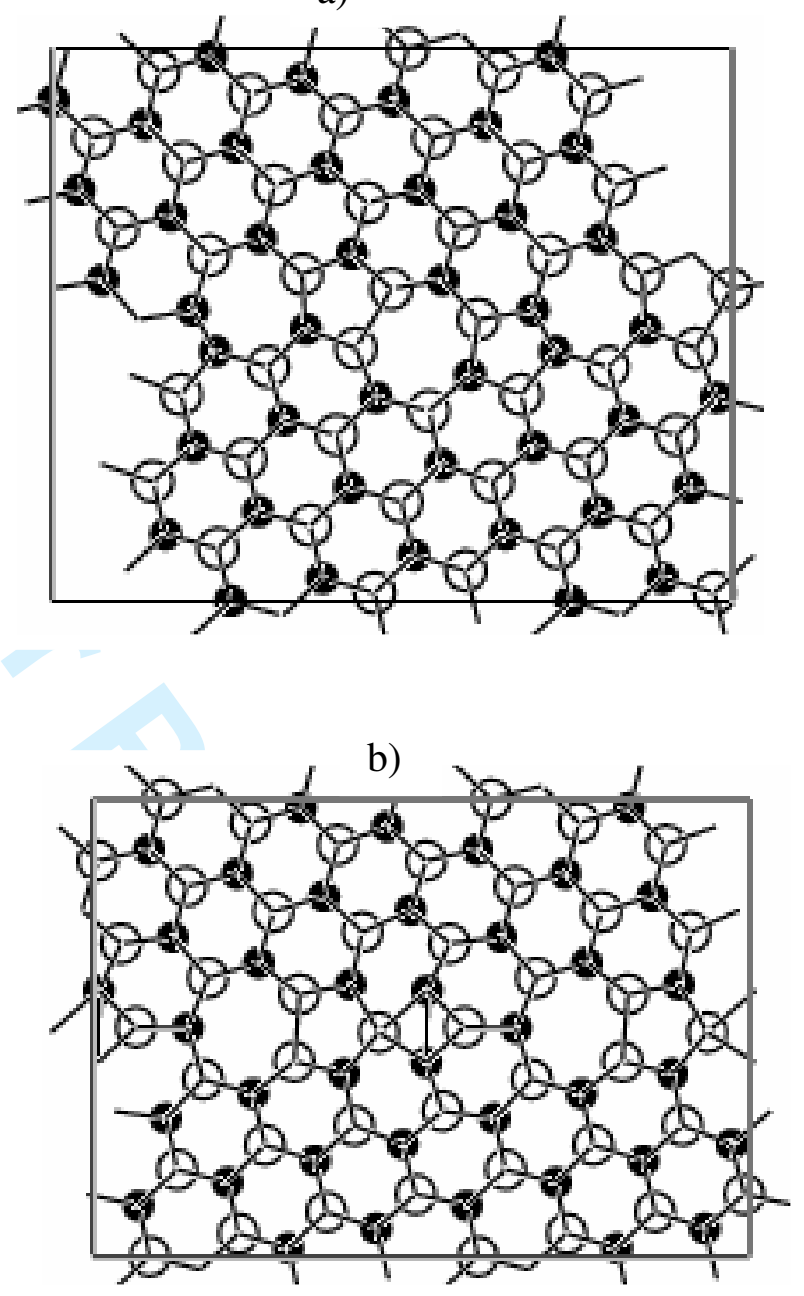

Fig.16 $(\Sigma 13)$ 



b)

a)



c)

Figure 17 


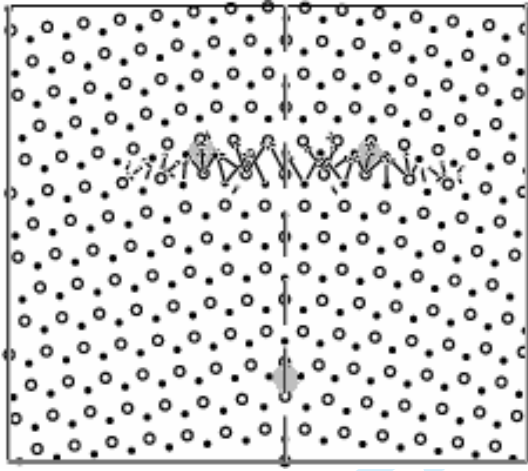

a)

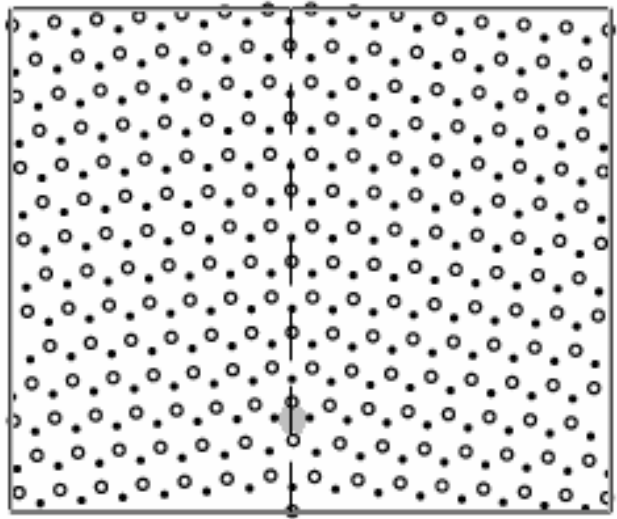

c)

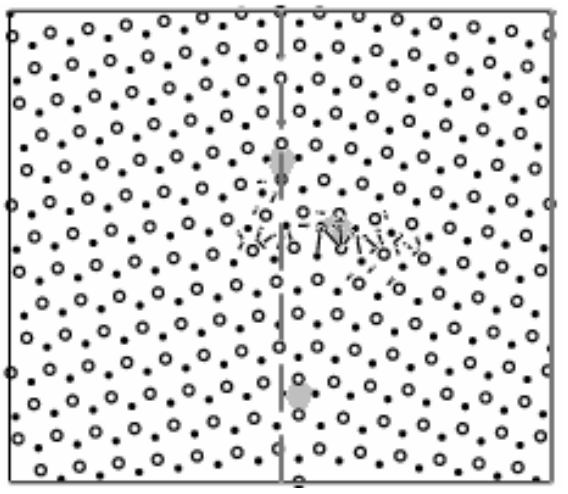

b)

Figure 18 




a)

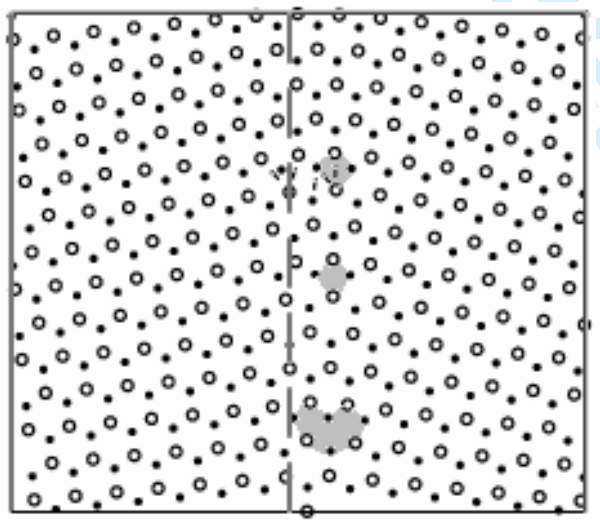

c)

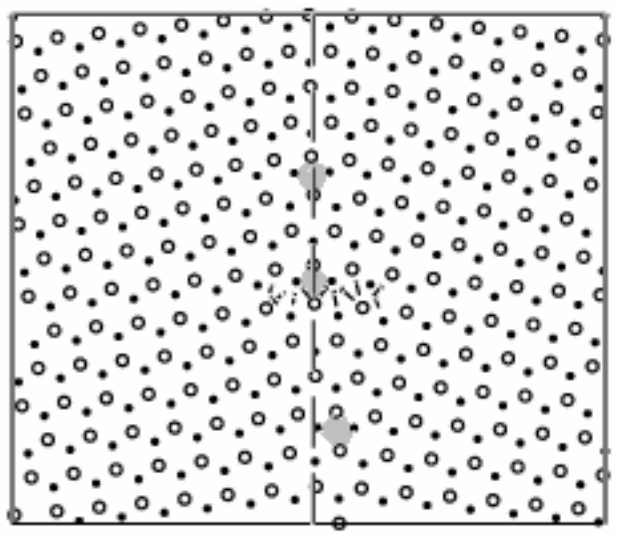

b)

Figure 19 
a)

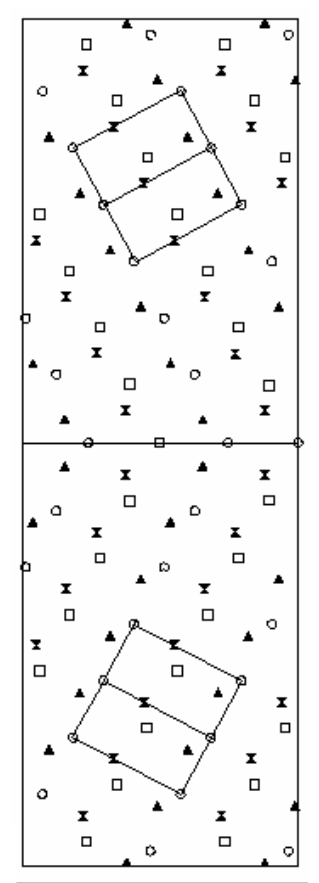

b)

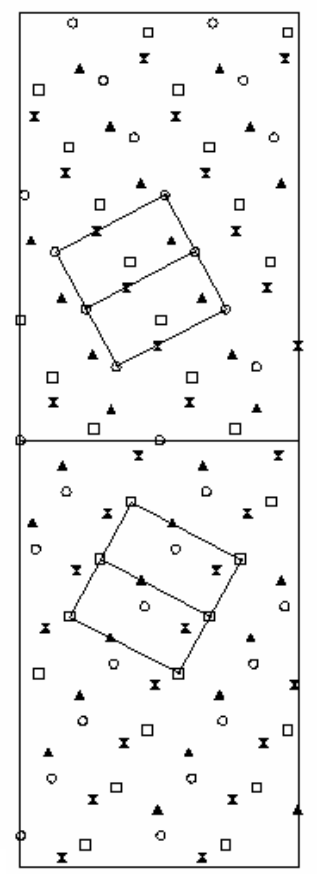

Figure 20

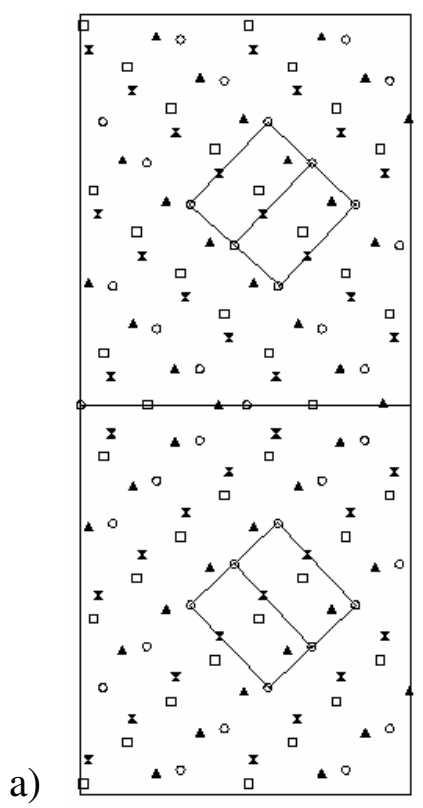

b)

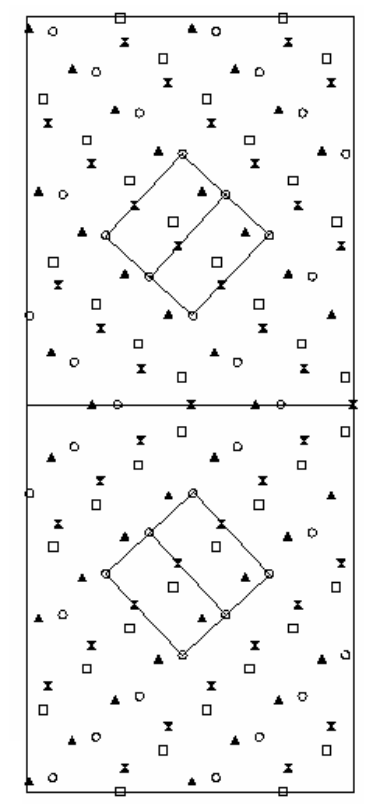

c)

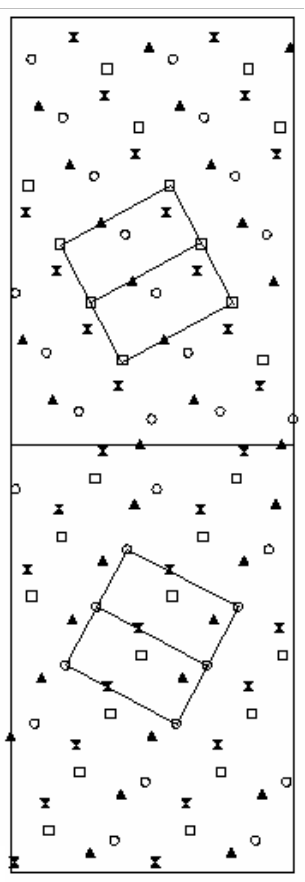
tand

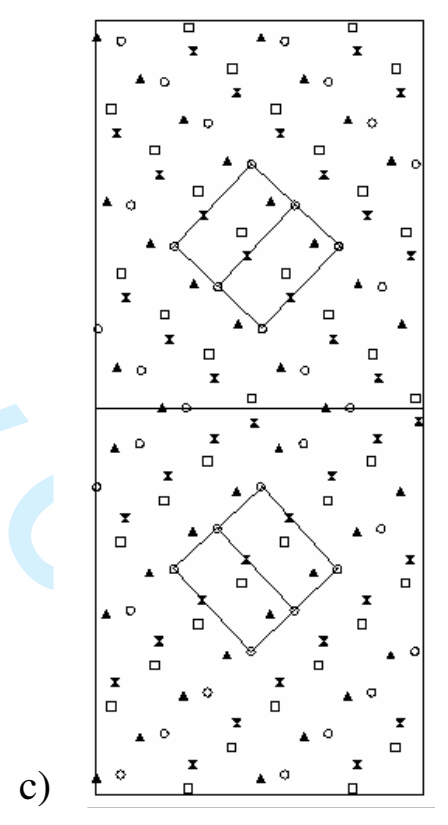

Figure 21 


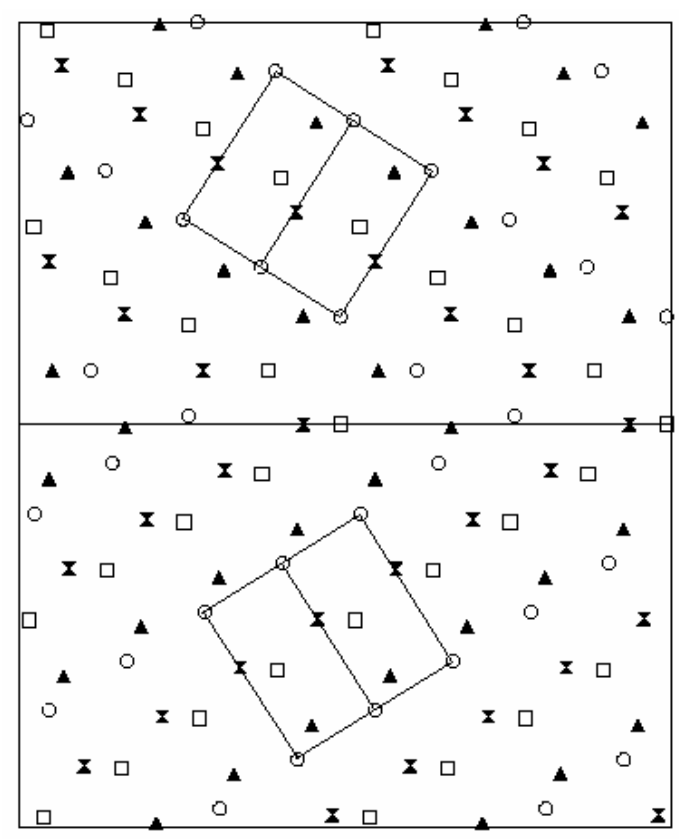

Figure 22 